\title{
An Enantioselective Total Synthesis of (-)-Candelalide A, a Novel Blocker of the Voltage-Gated Potassium Channel Kv1.3 for an Immunosuppressive agent
}

- Supporting Information \#1 -

Experimental Procedures

Kazuhiro Watanabe, ${ }^{\dagger}$ Katsuhiko Iwasaki, ${ }^{\ddagger}$ Toshiaki Abe, ${ }^{\ddagger}$ Munenori Inoue, ${ }^{\prime}$ Kōichi Ohkubo, ${ }^{\dagger}$ Takeyuki Suzuki, ${ }^{\dagger}$ and Tadashi Katoh ${ }^{* \dagger}$

${ }^{\dagger}$ Tohoku Pharmaceutical University, 4-4-1 Komatsushima, Aoba-ku, Sendai 981-8558, Japan, Department of Electronic Chemistry, Tokyo Institute of Technology, Nagatsuta, Yokohama 226-8502, Japan, ${ }^{\#}$ Sagami Chemical Research Center, 2743-1 Hayakawa, Ayase, Kanagawa 252-1193, Japan

E-mail: katoh@tohoku-pharm.ac.jp 


\section{General Procedure.}

All reactions involving air- and moisture-sensitive reagents were carried out using oven dried glassware and standard syringe-septum cap techniques. Routine monitorings of reaction were carried out using glass-supported Merck silica gel 60 $\mathrm{F}_{254}$ TLC plates. Flash column chromatography was performed on Kanto Chemical Silica Gel 60N (spherical, neutral 40-50 $\mu \mathrm{m})$ with the solvents indicated.

\section{Materials.}

All solvents and reagents were used as supplied with following exceptions. Tetrahydrofuran (THF) and diethyl ether were freshly distilled from sodium/benzophenone under argon. Toluene was distilled from sodium under argon. $N, N-$ Dimethylformamide (DMF), dichloromethane, acetonitrile, pyridine, and hexane were distilled from calcium hydride under argon.

\section{Instrumentation.}

Measurements of optical rotations were performed with a JASCO P-1020 automatic digital polarimeter. Melting points were taken on a Yanaco MP-3 micro melting point apparatus and are uncorrected. ${ }^{1} \mathrm{H}$ and ${ }^{13} \mathrm{C}$ NMR spectra were measured with a JEOL JNM-EX270 (270 MHz), a Brucker DRX-500 (500 MHz), or a JEOL JNM-LA600 (600 MHz) spectrometer. Chemical shifts were expressed in ppm using tetramethylsilane $(\delta=0)$ as an internal standard. The following abbreviations are used: singlet (s), doublet (d), triplet (t), multiplet (m), and broad (br). Infrared (IR) spectral measurements were carried out with a JASCO FT/IR-5300 spectrometer. Low-resolution mass (MS) spectra was measured on a Shimadzu GCMS-QP2010. High-resolution mass (HRMS) spectra was measured on a JEOL MStation JMS-700 mass spectrometer. Elemental analyses were performed with a Perkin Elmer 2400II apparatus. 
(4aS, 5S, 8aS)-5-Allyl-5,8a-dimethyldecahydronaphthalene-1,6-dione 1-ethyleneacetal (6).<smiles></smiles>

I
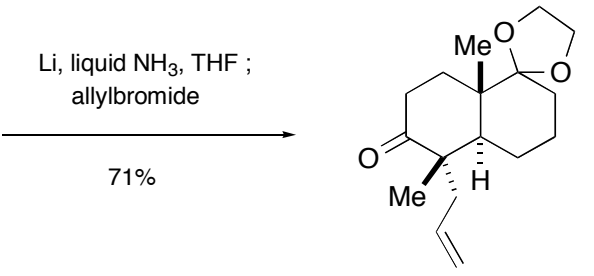

6

A solution of (8aS)-3,4,8,8a-tetrahydro-5,8a-dimethylnaphthalene-1,6-(2H,7H)-dione 1-ethyleneacetal (I) (4.10 g, 17 $\mathrm{mmol})$ in dry THF $(100 \mathrm{ml})$ was added dropwise to a stirred solution of lithium $(600 \mathrm{mg}, 87 \mathrm{mmol})$ in liquid ammonia (200 $\mathrm{ml}$ ) at $-78^{\circ} \mathrm{C}$ under argon. The resulting solution was allowed to warm at reflux of liquid ammonia for $1 \mathrm{~h}$, and then a solution of allylbromide $(7.50 \mathrm{ml}, 87 \mathrm{mmol})$ in dry THF $(10 \mathrm{ml})$ was added slowly. The mixture was allowed to stand at room temperature for $10 \mathrm{~h}$ in order to evaporate off ammonia. After addition of saturated aqueous ammonium chloride (30 ml), the resulting mixture was extracted with diethyl ether $(3 \times 100 \mathrm{ml})$. The combined extracts were washed with brine, and dried over $\mathrm{Na}_{2} \mathrm{SO}_{4}$. Concentration of the solvent in vacuo afforded a residue, which was purified by column chromatography (hexane-ethyl acetate, $20: 1)$ to give $6(3.42 \mathrm{~g}, 71 \%)$ as a colorless viscous liquid. $[\alpha]_{\mathrm{D}}{ }^{20}+28.0^{\circ}\left(c 0.71, \mathrm{CHCl}_{3}\right)\left[\mathrm{lit}^{4}+19^{\circ}(c\right.$ 2.15)]; ${ }^{1} \mathrm{H}$ NMR (500 MHz, $\left.\mathrm{CDCl}_{3}\right) \delta 1.04(3 \mathrm{H}, \mathrm{s}), 1.21(3 \mathrm{H}, \mathrm{s}), 1.35-1.50(2 \mathrm{H}, \mathrm{m}), 1.53-1.58(2 \mathrm{H}, \mathrm{m}), 1.61-1.72(3 \mathrm{H}, \mathrm{m})$, $1.94(1 \mathrm{H}, \mathrm{td}, J=5.8,13.2 \mathrm{~Hz}), 2.00(1 \mathrm{H}, \mathrm{dd}, J=8.9,13.9 \mathrm{~Hz}), 2.11-2.16(1 \mathrm{H}, \mathrm{m}), 2.34(1 \mathrm{H}, \mathrm{ddd}, J=3.5,5.8,16.0 \mathrm{~Hz}), 2.50-$ 2.59 (2H, m), 3.83-3.07 (4H, m), 4.96-5.05 (2H, m), 5.61-5.71 (1H, m); $\left.{ }^{13} \mathrm{C} \mathrm{NMR} \mathrm{(125} \mathrm{MHz,} \mathrm{CDCl}{ }_{3}\right) \delta 16.3,21.3,21.7$, 22.6, 28.8, 30.2, 35.0, 42.3, 42.6, 44.2, 50.9, 64.8, 65.2, 112.8, 117.3, 135.1, 215.7; IR (neat) 3075, 2048, 2880, 1703, 1640, $1441,1383,1337,1281,1236,1209,1184,1132,1100,1047,1017,951,910,864,727,563,478 \mathrm{~cm}^{-1}$; HREIMS $(m / z) \mathrm{calcd}$ for $\mathrm{C}_{17} \mathrm{H}_{26} \mathrm{O}_{3}\left(\mathrm{M}^{+}\right), 278.1882$, found 278.1870 .

(4aS,5S,6R,8aS)-6-Hydroxy-5-(3-hydroxypropyl)-5,8a-dimethyldecahydronaphthalen-1-one 1-ethyleneacetal (9).

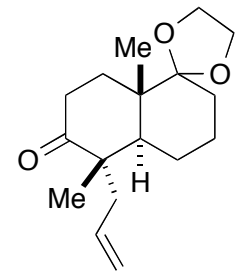

6

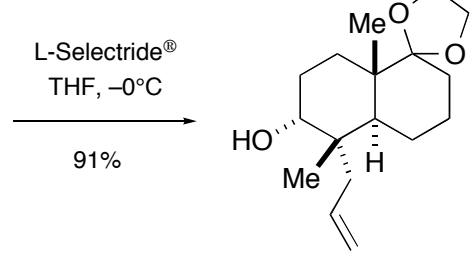

$\mathrm{BH}_{3} \cdot \mathrm{THF}, \mathrm{THF}, 0^{\circ} \mathrm{C}$; $3 \mathrm{M} \mathrm{NaOH}, 30 \%$ aq. $\mathrm{H}_{2} \mathrm{O}_{2}, 0^{\circ} \mathrm{C}$

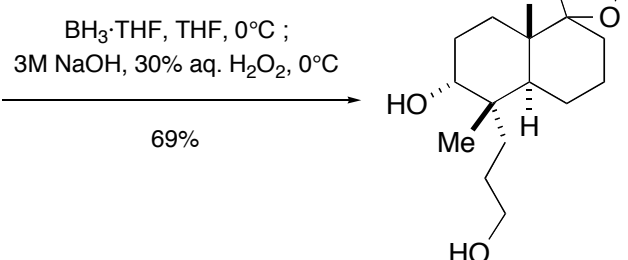

9

L-Selectride $^{\circledR}$ in THF (1.0 M solution, $4.40 \mathrm{ml}, 4.4 \mathrm{mmol}$ ) was added dropwise to a stirred solution of 6 (406 mg, 1.5 $\mathrm{mmol})$ in dry $\mathrm{THF}(10 \mathrm{ml})$ at $-10^{\circ} \mathrm{C}$ under argon. After $3 \mathrm{~h}$, the reaction was quenched with saturated aqueous ammonium chloride $(5.0 \mathrm{ml})$ at $-10^{\circ} \mathrm{C}$, and the resulting mixture was extracted with ethyl acetate $(3 \times 50 \mathrm{ml})$. The combined extracts were washed with brine $(2 \times 30 \mathrm{ml})$, then dried over $\mathrm{Na}_{2} \mathrm{SO}_{4}$. Concentration of the solvent in vacuo afforded a residue, which was purified by column chromatography (hexane-ethyl acetate, 10:1) to give $\mathbf{8}(372 \mathrm{mg}, 91 \%)$ as a colorless oil. This material was immediately used for the next reaction due to its instability.

A solution of borane-tetrahydrofuran complex in THF $(0.93 \mathrm{M}$ solution, $3.90 \mathrm{ml}, 3.7 \mathrm{mmol})$ was added dropwise to a 
stirred solution of $8(372 \mathrm{mg}, 1.3 \mathrm{mmol})$ in dry THF $(10 \mathrm{ml})$ at $0^{\circ} \mathrm{C}$ under argon. After $30 \mathrm{~min}$, water $(1.0 \mathrm{ml}), 3 \mathrm{M}$ sodium hydroxide $(2.0 \mathrm{ml})$, and $30 \%$ aqueous hydrogen peroxide $(2.0 \mathrm{ml})$ was added successively to the above mixture at $0^{\circ} \mathrm{C}$, and stirring was continued for $30 \mathrm{~min}$ at the same temperature. The reaction was quenched with brine $(5 \mathrm{ml})$ at $0^{\circ} \mathrm{C}$, and the resulting mixture was extracted with ethyl acetate $(3 \times 50 \mathrm{ml})$. The combined extracts were washed with brine $(2 \times 30 \mathrm{ml})$, then dried over $\mathrm{MgSO}_{4}$. Concentration of the solvent in vacuo afforded a residue, which was purified by column chromatography (hexane-ethyl acetate, $2: 1 \rightarrow 1: 2)$ to give $9(249 \mathrm{mg}, 69 \%)$ as a white amorphous solid. $[\alpha]_{\mathrm{D}}{ }^{20}-24.6^{\circ}(c 1.10$, $\left.\mathrm{CHCl}_{3}\right) ;{ }^{1} \mathrm{H}$ NMR $\left(500 \mathrm{MHz}, \mathrm{CDCl}_{3}\right) \delta 0.83(3 \mathrm{H}, \mathrm{s}), 1.10(3 \mathrm{H}, \mathrm{s}), 1.16-1.20(1 \mathrm{H}, \mathrm{m}), 1.24-1.32(1 \mathrm{H}, \mathrm{m}), 1.35-1.55(6 \mathrm{H}, \mathrm{m})$, 1.55-1.71 (6H, m), $1.79(1 \mathrm{H}, \mathrm{dd}, J=2.4,12.6 \mathrm{~Hz}), 1.88(2 \mathrm{H}, \mathrm{d}, J=9.8 \mathrm{~Hz}), 3.56-3.63(2 \mathrm{H}, \mathrm{m}), 3.64-3.70(1 \mathrm{H}, \mathrm{m}), 3.80-3.86$ $(1 \mathrm{H}, \mathrm{m}), 3.90-4.01(3 \mathrm{H}, \mathrm{m}) ;{ }^{13} \mathrm{C} \mathrm{NMR}\left(125 \mathrm{MHz}, \mathrm{CDCl}_{3}\right) \delta 16.7,18.6,19.9,22.8,22.9,24.6,25.4,30.2,35.0,39.1,42.7$, 43.1, 63.1, 64.6, 65.0, 71.3, 113.3; IR (neat) 3385, 2949, 2878, 1657, 1447, 1383, 1337, 1283, 1211, 1184, 1136, 1111, 1076, 993, 951, 909, 862, 797, 756, 666, 581, 513, $471 \mathrm{~cm}^{-1}$; HREIMS ( $\left.\mathrm{m} / \mathrm{z}\right)$ calcd for $\mathrm{C}_{17} \mathrm{H}_{30} \mathrm{O}_{4}\left(\mathrm{M}^{+}\right)$, 298.2144, found 298.2144 .

(4aS,5S,6R,8aS)-6-Hydroxy-5-(3-hydroxypropyl)-5,8a-dimethyldecahydronaphthalen-1-one (10).
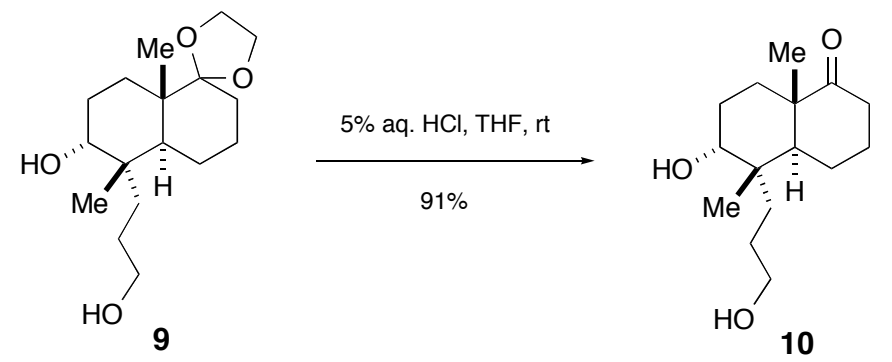

$5 \%$ Aqueous hydrochloric acid $(7 \mathrm{ml})$ was added dropwise to a stirred solution of 9 (240 $\mathrm{mg}, 0.81 \mathrm{mmol})$ in $\mathrm{THF}(6 \mathrm{ml})$ at room temperature. After $4 \mathrm{~h}, 3 \mathrm{M}$ sodium hydroxide $(3 \mathrm{ml})$ and saturated aqueous sodium hydrogen carbonate $(3 \mathrm{ml})$ were added slowly to the mixture at $0^{\circ} \mathrm{C}$. The resulting mixture was extracted with ethyl acetate $(4 \mathrm{x} 30 \mathrm{ml})$. The combined extracts were washed with saturated aqueous sodium hydrogen carbonate $(2 \times 25 \mathrm{ml})$ and brine $(2 \times 25 \mathrm{ml})$, then dried over $\mathrm{Na}_{2} \mathrm{SO}_{4}$. Concentration of the solvent in vacuo afforded a residue, which was purified by column chromatography (hexaneethyl acetate, $1: 1 \rightarrow 1: 2)$ to give $\mathbf{1 0}(186 \mathrm{mg}, 91 \%)$ as a white solid. Recrystallization from THF afforded white needles, mp $169-170^{\circ} \mathrm{C} ;[\alpha]_{\mathrm{D}}{ }^{20}-50.9^{\circ}\left(c 1.09, \mathrm{CHCl}_{3}\right) ;{ }^{1} \mathrm{H} \mathrm{NMR}\left(500 \mathrm{MHz}, \mathrm{CDCl}_{3}\right) \delta 0.92(3 \mathrm{H}, \mathrm{s}), 1.18(3 \mathrm{H}, \mathrm{s}), 1.39(1 \mathrm{H}, \mathrm{dt}, J=3.1,13.7$ $\mathrm{Hz}), 1.41-1.56(3 \mathrm{H}, \mathrm{m}), 1.58-1.71(7 \mathrm{H}, \mathrm{m}), 1.72-1.77(1 \mathrm{H}, \mathrm{m}), 1.90(1 \mathrm{H}, \mathrm{ddt}, J=2.4,3.5,14.4 \mathrm{~Hz}), 1.99(1 \mathrm{H}, \mathrm{dd}, J=3.5,13.7$ $\mathrm{Hz}), 2.02-2.10(1 \mathrm{H}, \mathrm{m}), 2.19-2.26(1 \mathrm{H}, \mathrm{m}), 2.58(1 \mathrm{H}, \mathrm{dt}, J=6.9,13.8 \mathrm{~Hz}), 3.58-3.65(2 \mathrm{H}, \mathrm{m}), 3.65-3.72(1 \mathrm{H}, \mathrm{m}) ;{ }^{13} \mathrm{C} \mathrm{NMR}$ $\left(125 \mathrm{MHz}, \mathrm{CDCl}_{3}\right) \delta 18.8,19.0,20.1,24.8,25.5,25.6,26.0,35.0,37.4,40.2,46.9,48.8,63.3,70.9,215.4$; FT-IR (KBr) 3387, 2959, 2934, 2880, 1696, 1451, 1433, 1385, 1306, 1242, 1117, 1055, 1015, 976, 961, 920, 822, 748, $556 \mathrm{~cm}^{-1}$; HREIMS (m/z) calcd for $\mathrm{C}_{15} \mathrm{H}_{26} \mathrm{O}_{3}\left(\mathrm{M}^{+}\right), 254.1882$, found 254.1887; Anal calcd for $\mathrm{C}_{15} \mathrm{H}_{26} \mathrm{O}_{3}: \mathrm{C}, 70.83 ; \mathrm{H}, 10.30$, found C, $70.85 ; \mathrm{H}, 9.89$.

(4aS,5S,6R,8aS)-5-(3-tert-Butyldimethylsilyloxypropyl)-6-hydroxy-5,8a-dimethyldecahydronaphthalen-1-one (11).
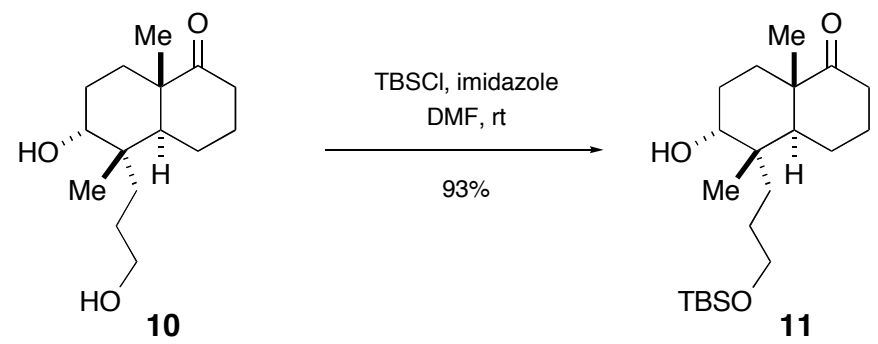
tert-Butyldimethylsilyl chloride (TBSCl) $(213 \mathrm{mg}, 1.4 \mathrm{mmol})$ was added to a stirred solution of $\mathbf{1 0}(180 \mathrm{mg}, 0.71 \mathrm{mmol}) \mathrm{in}$ dry DMF (4 ml) containing imidazole $(120 \mathrm{mg}, 1.8 \mathrm{mmol})$ at room temperature. After $14 \mathrm{~h}$, the reaction was quenched with saturated aqueous ammonium chloride $(3 \mathrm{ml})$ at $0^{\circ} \mathrm{C}$, and the resulting mixture was extracted with diethyl ether $(3 \mathrm{x} 40 \mathrm{ml})$. The combined extracts were washed successively with $2 \%$ aqueous hydrochloric acid ( $2 \times 25 \mathrm{ml})$, saturated aqueous sodium hydrogen carbonate $(2 \times 25 \mathrm{ml})$, and brine $(2 \times 25 \mathrm{ml})$, then dried over $\mathrm{Na}_{2} \mathrm{SO}_{4}$. Concentration of the solvent in vacuo afforded a residue, which was purified by column chromatography (hexane-ethyl acetate, 10:1) to give 11 (243 $\mathrm{mg}$, $93 \%)$ as a white solid. Recrystallization from hexane-diethyl ether (10:1) afforded white needles, mp $75-77^{\circ} \mathrm{C} ;[\alpha]_{\mathrm{D}}{ }^{20}-27.8^{\circ}(c 1.31$, $\left.\mathrm{CHCl}_{3}\right) ;{ }^{1} \mathrm{H} \mathrm{NMR}\left(500 \mathrm{MHz}, \mathrm{CDCl}_{3}\right) \delta 0.06(6 \mathrm{H}, \mathrm{s}), 0.90(9 \mathrm{H}, \mathrm{s}), 0.91(3 \mathrm{H}, \mathrm{s}), 1.17$ (3H, s), 1.29-1.39 (2H, m), $1.42-1.49$ $(1 \mathrm{H}, \mathrm{m}), 1.50-1.54(2 \mathrm{H}, \mathrm{m}), 1.59-1.65(2 \mathrm{H}, \mathrm{m}), 1.65-1.74(3 \mathrm{H}, \mathrm{m}), 1.81-1.90(1 \mathrm{H}, \mathrm{m}), 1.93(1 \mathrm{H}, \mathrm{d}, J=3.4 \mathrm{~Hz}), 1.97-2.09$ $(2 \mathrm{H}, \mathrm{m}), 2.18-2.26(1 \mathrm{H}, \mathrm{m}), 2.57(1 \mathrm{H}, \mathrm{dt}, J=6.9,13.7 \mathrm{~Hz}), 3.52-3.66(3 \mathrm{H}, \mathrm{m}) ;{ }^{13} \mathrm{C} \mathrm{NMR}\left(125 \mathrm{MHz}, \mathrm{CDCl}_{3}\right) \delta-5.2,-5.3,18.4$, 18.9, 19.1, 20.2 , 24.7, 25.7, 25.8, 26.0 (3 carbons), 34.9, 37.5, 40.3, 46.8, 48.8, 63.8, 71.0, 77.2, 215.2; IR (KBr) 3474, 2953, 2930, 2859, 1698, 1472, 1385, 1256, 1209, 1167, 1063, 1019, 966, 891, 837, 775, 735, 662, 559, 498, 421 cm ${ }^{-1}$; HREIMS $(\mathrm{m} / z)$ calcd for $\mathrm{C}_{21} \mathrm{H}_{40} \mathrm{O}_{3} \mathrm{Si}\left(\mathrm{M}^{+}\right)$, 368.2747, found 368.2748; Anal calcd for $\mathrm{C}_{21} \mathrm{H}_{40} \mathrm{O}_{3} \mathrm{Si}$ : C, 68.42; H, 10.94, found C, 68.42; H, 10.94 .

\section{(4aS,5S,6R,8aS)-5-(3-tert-Butyldimethylsilyloxypropyl)-6-triethylsilyloxy-5,8a-dimethyldecahydronaphthalen-1-one} (12).

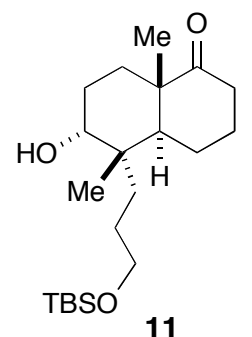

11

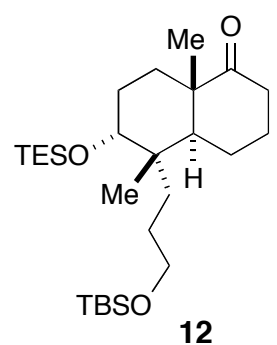

12

Triethylsilyl trifluoromethanesulfonate (TESOTf) $(0.443 \mathrm{ml}, 1.9 \mathrm{mmol})$ was added dropwise to a stirred solution of 11 (230 $\mathrm{mg}, 0.63 \mathrm{mmol})$ in dry dichloromethane $(6 \mathrm{ml})$ containing 2,6-lutidine $(0.239 \mathrm{ml}, 2.1 \mathrm{mmol})$ at $0^{\circ} \mathrm{C}$ under argon. After $30 \mathrm{~min}$, the reaction was quenched with saturated aqueous ammonium chloride $(3 \mathrm{ml})$ at $0^{\circ} \mathrm{C}$, and the resulting mixture was extracted with diethyl ether $(3 \times 30 \mathrm{ml})$. The combined extracts were washed successively with $2 \%$ aqueous hydrochloric acid $(2 \times 20$ $\mathrm{ml})$, saturated aqueous sodium hydrogen carbonate $(2 \times 20 \mathrm{ml})$, and brine $(2 \times 20 \mathrm{ml})$, then dried over $\mathrm{Na}_{2} \mathrm{SO}_{4}$. Concentration of the solvent in vacuo afforded a residue, which was purified by column chromatography (hexane-ethyl acetate, 50:1) to give $12(247 \mathrm{mg}, 82 \%)$ as a colorless viscous liquid. $[\alpha]_{\mathrm{D}}{ }^{20}-19.4^{\circ}\left(c 1.34, \mathrm{CHCl}_{3}\right) ;{ }^{1} \mathrm{H} \mathrm{NMR}\left(500 \mathrm{MHz}, \mathrm{CDCl}_{3}\right): \delta 0.04(6 \mathrm{H}$, s), $0.61(6 \mathrm{H}, \mathrm{dq}, J=5.3,7.9 \mathrm{~Hz}), 0.88(3 \mathrm{H}, \mathrm{s}), 0.89(9 \mathrm{H}, \mathrm{s}), 0.95(9 \mathrm{H}, \mathrm{t}, J=7.9 \mathrm{~Hz}), 1.16(3 \mathrm{H}, \mathrm{s}), 1.23-1.33(4 \mathrm{H}, \mathrm{m}), 1.44-1.53$ $(2 \mathrm{H}, \mathrm{m}), 1.58-1.66(4 \mathrm{H}, \mathrm{m}), 1.79(1 \mathrm{H}, \mathrm{ddt}, J=2.1,3.4,14.4 \mathrm{~Hz}), 1.97-2.07(2 \mathrm{H}, \mathrm{m}), 2.17-2.24(1 \mathrm{H}, \mathrm{m}), 2.50-2.61(1 \mathrm{H}, \mathrm{m})$, 3.46-3.54 (1H, m), 3.55-3.62 (2H, m); $\left.{ }^{13} \mathrm{C} \mathrm{NMR} \mathrm{(125MHz,} \mathrm{CDCl}_{3}\right)$ : $\delta$-5.2, -5.3, 5.4 (3 carbons), 7.1 (3 carbons), 18.3, 19.2, 19.3, 20.1 , 25.3, 25.8, 25.9, 26.0 (3 carbons), 26.7, 35.4, 37.5, 40.8, 46.4, 48.8, 64.1, 72.9, 215.6; IR (neat) 2955, 2878, 1711, $1462,1385,1362,1306,1252,1096,1007,963,941,909,837,775,739,687,559 \mathrm{~cm}^{-1}$; HREIMS (m/z) calcd for $\mathrm{C}_{27} \mathrm{H}_{54} \mathrm{O}_{3} \mathrm{Si}_{2}\left(\mathrm{M}^{+}\right), 482.3611$, found 482.3608 . 
(Z)-(4aS,5S,6R,8aS)-5-(3-tert-Butyldimethylsilyloxypropyl)-6-triethylsilyloxy-2-hydroxymethylene-5,8a-dimethyldecahydronaphthalen-1-one (13).
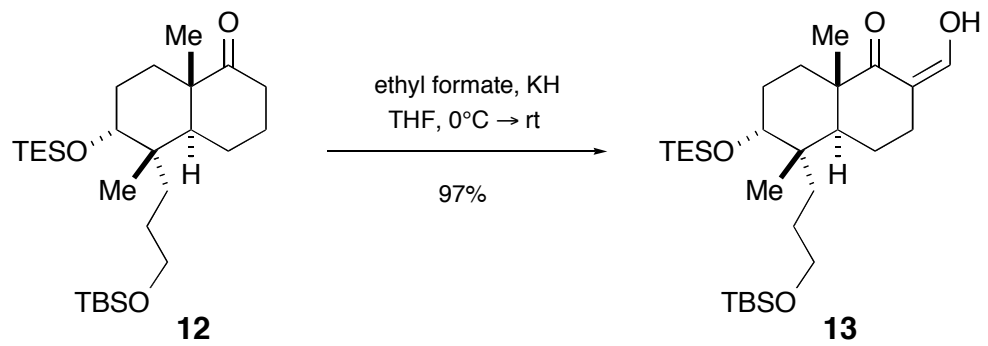

A solution of $12(230 \mathrm{mg}, 0.48 \mathrm{mmol})$ in dry THF $(6 \mathrm{ml})$ containing ethyl formate $(0.462 \mathrm{ml}, 5.7 \mathrm{mmol})$ was added to a stirred suspension of potassium hydride $(286 \mathrm{mg}, 7.2 \mathrm{mmol})$ in dry THF $(6 \mathrm{ml})$ at $0^{\circ} \mathrm{C}$ under argon, and stirring was continued for $30 \mathrm{~min}$ at room temperature. The reaction was quenched with saturated aqueous ammonium chloride (5 ml) at $0^{\circ} \mathrm{C}$, and the resulting mixture was extracted with diethyl ether $(3 \times 30 \mathrm{ml})$. The combined extracts were washed with brine $(2$ x $20 \mathrm{ml}$ ), then dried over $\mathrm{Na}_{2} \mathrm{SO}_{4}$. Concentration of the solvent in vacuo afforded a residue, which was purified by column chromatography (hexane-ethyl acetate, $10: 1)$ to give $\mathbf{1 3}(236 \mathrm{mg}, 97 \%)$ as a pale yellow viscous liquid. $[\alpha]_{\mathrm{D}}^{20}+0.4^{\circ}(c 1.46$, $\left.\mathrm{CHCl}_{3}\right) ;{ }^{1} \mathrm{H}$ NMR $\left(500 \mathrm{MHz}, \mathrm{CDCl}_{3}\right) \delta 0.05(6 \mathrm{H}, \mathrm{s}), 0.61(6 \mathrm{H}, \mathrm{dq}, J=2.3,7.9 \mathrm{~Hz}), 0.83(3 \mathrm{H}, \mathrm{s}), 0.89(9 \mathrm{H}, \mathrm{s}), 0.95(9 \mathrm{H}, \mathrm{t}, J=7.9$ $\mathrm{Hz}), 1.21(3 \mathrm{H}, \mathrm{s}), 1.27-1.34(3 \mathrm{H}, \mathrm{m}), 1.41-1.53$ (3H, m), 1.57-1.67 (2H, m), 1.68-1.74 (1H, m), 1.80-1.88 (2H, m), 2.32 (1H, ddd, $J=7.0,11.5,15.2 \mathrm{~Hz}), 2.44(1 \mathrm{H}, \mathrm{ddd}, J=1.8,6.6,15.2 \mathrm{~Hz}), 3.49-3.56(1 \mathrm{H}, \mathrm{m}), 3.56-3.64(2 \mathrm{H}, \mathrm{m}), 8.40(1 \mathrm{H}, \mathrm{d}, J=4.5$ $\mathrm{Hz}), 14.65(1 \mathrm{H}, \mathrm{d}, J=4.5 \mathrm{~Hz}) ;{ }^{13} \mathrm{C}$ NMR $\left(125 \mathrm{MHz}, \mathrm{CDCl}_{3}\right) \delta-5.2,-5.3,5.4$ (3 carbons), 7.2 (3 carbons), 17.8, 18.4, 19.0, 20.6, 23.1, 25.6, 26.0 (3 carbons), 26.6, 35.0, 40.2, 42.0, 42.1, 48.7, 64.1, 72.9, 106.0, 184.5, 195.5; IR (neat) 2955, 2879, $1705,1640,1586,1462,1412,1385,1362,1334,1254,1100,1057,1009,966,936,907,837,797,775,739,687,556 \mathrm{~cm}^{-1}$; HREIMS $(\mathrm{m} / \mathrm{z})$ calcd for $\mathrm{C}_{28} \mathrm{H}_{54} \mathrm{O}_{4} \mathrm{Si}_{2}\left(\mathrm{M}^{+}\right), 510.3561$, found 510.3563 .

(Z)-(4aS,5S,6R,8aS)-5-(3-tert-Butyldimethylsilyloxypropyl)-2-(ethoxyethoxyethan-1-yl)oxymethylene-6-triethylsilyloxy5,8a-dimethyldecahydronaphthalen-1-one (14).
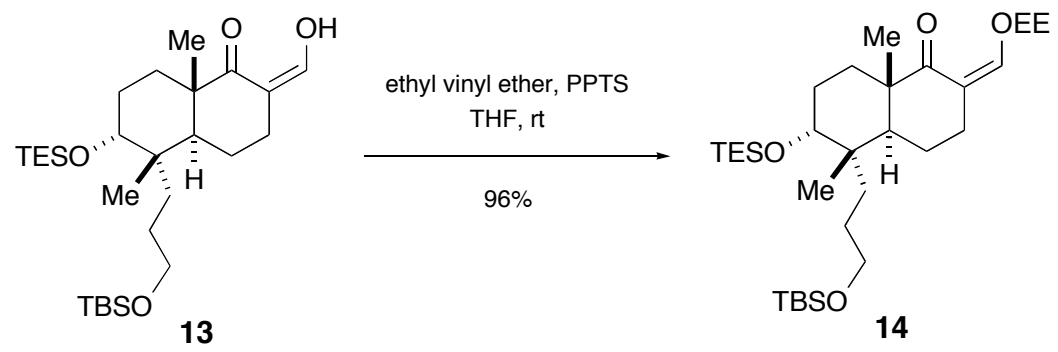

Pyridinium $p$-toluenesulfonate (PPTS) $(11.0 \mathrm{mg}, 44 \mu \mathrm{mol})$ was added to a stirred solution of 13 (224 mg, $0.44 \mathrm{mmol})$ in dry THF $(6 \mathrm{ml})$ containing ethyl vinyl ether $(0.723 \mathrm{ml}, 8.8 \mathrm{mmol})$ at room temperature. After $1.5 \mathrm{~h}$, the reaction was quenched with saturated aqueous sodium hydrogen carbonate $(4 \mathrm{ml})$ at $0^{\circ} \mathrm{C}$, and the resulting mixture was extracted with ethyl acetate $(2 \times 50 \mathrm{ml})$. The combined extracts were washed with brine $(2 \times 20 \mathrm{ml})$, then dried over $\mathrm{Na}_{2} \mathrm{SO}_{4}$. Concentration of the solvent in vacuo afforded a residue, which was purified by column chromatography (hexane-ethyl acetate, 20:1 $\rightarrow 10: 1$ ) to give 14 (1:1 diastereomeric mixture caused by the EE group) $(245 \mathrm{mg}, 96 \%)$ as a pale yellow viscous liquid. $[\alpha]_{\mathrm{D}}{ }^{20}-16.8^{\circ}$ (c 1.28, $\left.\mathrm{CHCl}_{3}\right) ;{ }^{1} \mathrm{H} \mathrm{NMR}\left(500 \mathrm{MHz}, \mathrm{CDCl}_{3}\right) \delta 0.05(6 \mathrm{H}, \mathrm{s}), 0.56-0.64(6 \mathrm{H}, \mathrm{m}), 0.85(3 \mathrm{H}, \mathrm{s}), 0.90(9 \mathrm{H}, \mathrm{s}), 0.94(9 \mathrm{H}, \mathrm{t}, J=7.9$ 
$\mathrm{Hz}), 1.12(3 \mathrm{H}, \mathrm{s}), 1.21(3 \mathrm{H}, \mathrm{t}, J=7.1 \mathrm{~Hz}), 1.30-1.36(2 \mathrm{H}, \mathrm{m}), 1.42(3 / 2 \mathrm{H}, \mathrm{d}, J=5.2 \mathrm{~Hz}), 1.43(3 / 2 \mathrm{H}, \mathrm{d}, J=5.2 \mathrm{~Hz}), 1.46-1.53$ $(2 \mathrm{H}, \mathrm{m}), 1.55-1.65(3 \mathrm{H}, \mathrm{m}), 1.70-1.90(4 \mathrm{H}, \mathrm{m}), 2.20-2.30(1 \mathrm{H}, \mathrm{m}), 2.64-2.72(1 \mathrm{H}, \mathrm{m}), 3.45-3.55(2 \mathrm{H}, \mathrm{m}), 3.56-3.63(2 \mathrm{H}$, m), 3.68-3.75 $(1 \mathrm{H}, \mathrm{m}), 5.07(1 \mathrm{H}$, quint, $J=5.2 \mathrm{~Hz}), 7.40-7.44(1 \mathrm{H}, \mathrm{m}) ;{ }^{13} \mathrm{C} \mathrm{NMR}\left(125 \mathrm{MHz}, \mathrm{CDCl}_{3}\right) \delta-5.2,-5.3,5.4(3$ carbons), 7.2 (3 carbons), 14.9 (1/2 carbon), 15.0 (1/2 carbon), 18.0, 18.4, 19.4, 19.6 (1/2 carbon), 19.7 (1/2 carbon), 21.1, 22.2 (1/2 carbon), 22.3 (1/2 carbon), 25.6, 26.0 (3 carbon), 26.7, 26.8, 35.0, 40.6, 42.1 (1/2 carbon), 42.2 (1/2 carbon), 46.4, 63.6 (1/2 carbon), 63.7 (1/2 carbon), 64.2, 73.0, 103.5 (1/2 carbon), 103.7 (1/2 carbon), 114.0 (1/2 carbon), 114.1 (1/2 carbon), 151.3 (1/2 carbon), 151.4 (1/2 carbon), 206.5 (1/2 carbon), 206.6 (1/2 carbon); IR (neat) 2955, 2880, 1680, 1599, $1462,1385,1345,1254,1209,1101,1046,1005,972,882,837,775,739,689 \mathrm{~cm}^{-1}$; HREIMS $(\mathrm{m} / z)$ calcd for $\mathrm{C}_{32} \mathrm{H}_{62} \mathrm{O}_{5} \mathrm{Si}_{2}$ $\left(\mathrm{M}^{+}\right), 582.4136$, found 582.4138.

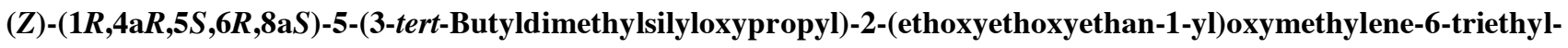
silyloxy-5,8a-dimethyldecahydronaphthalen-1-ol (15).
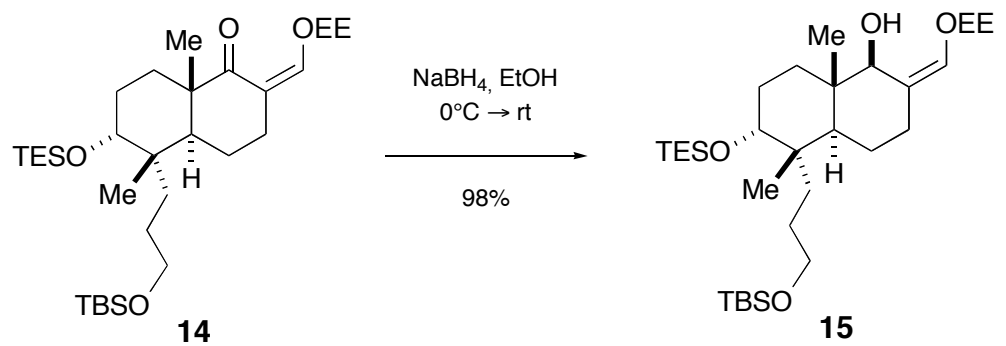

Sodium borohydride $(59.0 \mathrm{mg}, 1.6 \mathrm{mmol})$ was added in small portions to a stirred solution of 14 (182 $\mathrm{mg}, 0.31 \mathrm{mmol})$ in ethyl alcohol $(6 \mathrm{ml})$ at $0^{\circ} \mathrm{C}$, and stirring was continued for $1.5 \mathrm{~h}$ at room temperature. The reaction was quenched with $10 \%$ aqueous acetic acid $(2 \mathrm{ml})$ at $0^{\circ} \mathrm{C}$, and the resulting mixture was extracted with ethyl acetate $(3 \times 35 \mathrm{ml})$. The combined extracts were washed with saturated aqueous sodium hydrogen carbonate $(2 \times 20 \mathrm{ml})$ and brine $(2 \times 20 \mathrm{ml})$, then dried over $\mathrm{Na}_{2} \mathrm{SO}_{4}$. Concentration of the solvent in vacuo afforded a residue, which was purified by column chromatography (hexaneethyl acetate, 10:1) to give 15 (1:1 diastereomeric mixture caused by the EE group) (180 $\mathrm{mg}, 98 \%)$ as a colorless viscous liquid. $[\alpha]_{\mathrm{D}}{ }^{20}-16.2^{\circ}\left(c 1.50, \mathrm{CHCl}_{3}\right) ;{ }^{1} \mathrm{H} \mathrm{NMR}\left(500 \mathrm{MHz}, \mathrm{CDCl}_{3}\right) \delta 0.04(6 \mathrm{H}, \mathrm{s}), 0.58-0.65(6 \mathrm{H}, \mathrm{m}), 0.77(3 \mathrm{H}, \mathrm{s}), 0.78(3 \mathrm{H}, \mathrm{s})$, $0.89(9 \mathrm{H}, \mathrm{s}), 0.97(9 \mathrm{H}, \mathrm{t}, J=7.9 \mathrm{~Hz}), 1.20(3 / 2 \mathrm{H}, \mathrm{t}, J=7.1 \mathrm{~Hz}), 1.21(3 / 2 \mathrm{H}, \mathrm{t}, J=7.1 \mathrm{~Hz}), 1.17-1.32(4 \mathrm{H}, \mathrm{m}), 1.36(3 \mathrm{H}, \mathrm{d}, J=5.3$ $\mathrm{Hz}), 1.40(1 \mathrm{H}, \mathrm{dd}, J=1.0,5.2 \mathrm{~Hz}), 1.43-1.52(5 \mathrm{H}, \mathrm{m}), 1.57-1.68(2 \mathrm{H}, \mathrm{m}), 1.75-1.85(1 \mathrm{H}, \mathrm{m}), 2.90$ (1H, dd, $J=2.9,14.0 \mathrm{~Hz})$, 3.45-3.54 (2H, m), 3.55-3.62 (2H, m), 3.70-3.77 (2H, m), 4.86-4.94 (1H, m), 6.21-6.27 (1H, m); ${ }^{13} \mathrm{C} \mathrm{NMR} \mathrm{(125MHz,}$ $\left.\mathrm{CDCl}_{3}\right) \delta-5.2,-5.3,5.4$ (3 carbons), 7.2 (3 carbons), 13.0 (1/2 carbon), 13.1 (1/2 carbon), 15.1 (1/2 carbon), 15.2 (1/2 carbon), 18.4, 19.0, 20.5 (1/2 carbon), 20.6 (1/2 carbon), 21.0 (1/2 carbon), 21.1 (1/2 carbon), 24.3, 25.9, 26.0 (3 carbons), 26.8, 30.1 (1/2 carbon), 30.2 (1/2 carbon), 35.3, 39.7, 40.5 (1/2 carbon), 40.6 (1/2 carbon), 45.6, 62.3 (1/2 carbon), 62.4 (1/2 carbon), 64.3, 73.4, 80.8, 100.8 (1/2 carbon), 100.9 (1/2 carbon), 119.0 (1/2 carbon), 119.1 (1/2 carbon), 133.4 (1/2 carbon), 133.7 (1/2 carbon); IR (neat) 2955, 2878, 1686, 1462, 1383, 1341, 1254, 1086, 1007, 965, 936, 837, 808, 775, 739 cm ${ }^{-1}$; HREIMS $(m / z)$ calcd for $\mathrm{C}_{32} \mathrm{H}_{64} \mathrm{O}_{5} \mathrm{Si}_{2}\left(\mathrm{M}^{+}\right), 584.4292$, found 584.4291. 
(4aR,5S,6R,8aR)-5-(3-tert-Butyldimethylsilyloxypropyl)-5,8a-dimethyl-6-triethylsilyloxy-3,4,4a,5,6,7,8,8a-octahydronaphthalene-2-carbaldehyde (16).
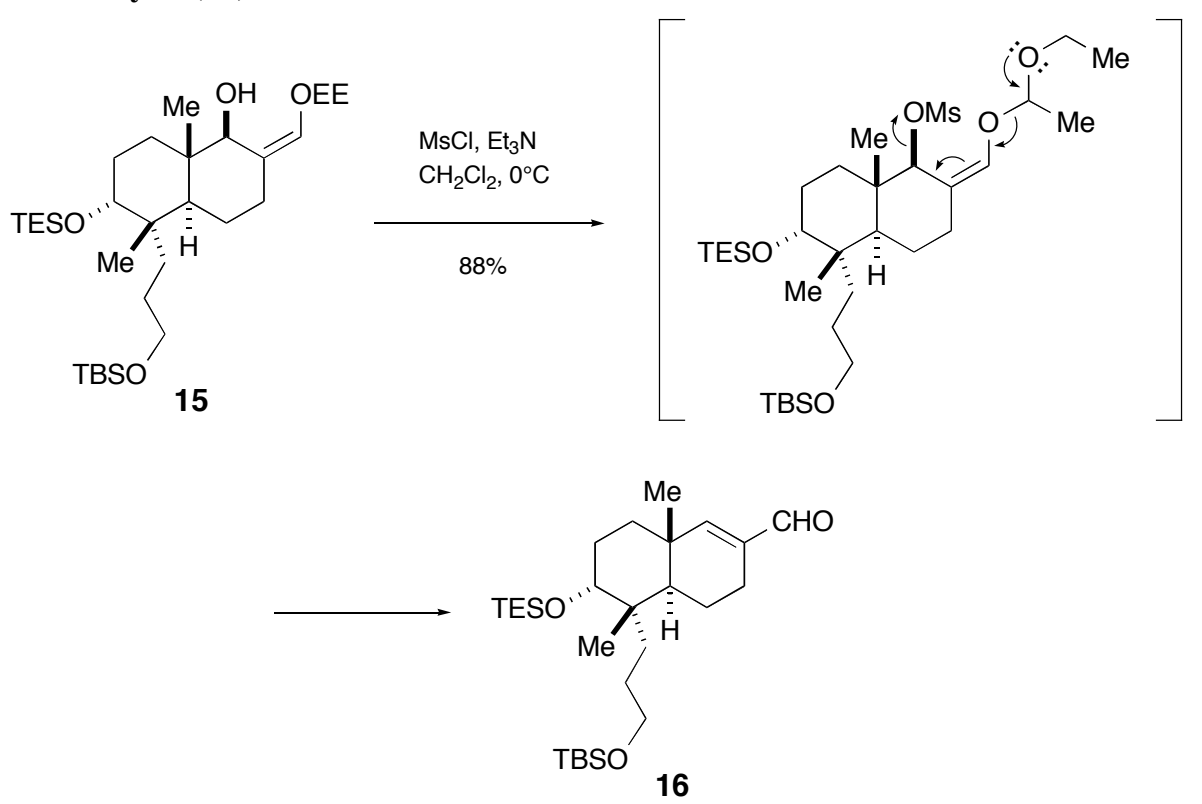

Methanesulfonyl chloride $(0.590 \mathrm{ml}, 7.5 \mathrm{mmol})$ was added dropwise to a stirred solution of 15 (436 $\mathrm{mg}, 0.75 \mathrm{mmol})$ in dry dichloromethane $(8.0 \mathrm{ml})$ containing triethylamine $(1.25 \mathrm{ml}, 8.9 \mathrm{mmol})$ at $0^{\circ} \mathrm{C}$ under argon. After $30 \mathrm{~min}$, the reaction was quenched with saturated aqueous ammonium chloride $(5 \mathrm{ml})$ at $0^{\circ} \mathrm{C}$, and the resulting mixture was extracted with ethyl acetate $(3 \times 40 \mathrm{ml})$. The combined extracts were washed with brine $(2 \times 30 \mathrm{ml})$, then dried over $\mathrm{Na}_{2} \mathrm{SO}_{4}$. Concentration of the solvent in vacuo afforded a residue, which was purified by column chromatography (hexane-ethyl acetate, 20:1) to give $\mathbf{1 6}$ (324 mg, 88\%) as a colorless viscous liquid. $[\alpha]_{\mathrm{D}}{ }^{20}+2.1^{\circ}\left(\mathrm{c} 1.36, \mathrm{CHCl}_{3}\right) ;{ }^{1} \mathrm{H} \mathrm{NMR}\left(500 \mathrm{MHz}, \mathrm{CDCl}_{3}\right) \delta 0.05(6 \mathrm{H}, \mathrm{s}), 0.61$ $(6 \mathrm{H}, \mathrm{q}, J=7.9 \mathrm{~Hz}), 0.82(3 \mathrm{H}, \mathrm{s}), 0.90(9 \mathrm{H}, \mathrm{s}), 0.95(9 \mathrm{H}, \mathrm{t}, J=7.9 \mathrm{~Hz}), 1.06(3 \mathrm{H}, \mathrm{s}), 1.24-1.33(3 \mathrm{H}, \mathrm{m}), 1.33-1.44(1 \mathrm{H}, \mathrm{m})$, $1.45-1.55(2 \mathrm{H}, \mathrm{m}), 1.56-1.62(1 \mathrm{H}, \mathrm{m}), 1.64(1 \mathrm{H}, \mathrm{dd}, J=1.7,12.8 \mathrm{~Hz}), 1.72-1.86(2 \mathrm{H}, \mathrm{m}), 1.90-1.99(1 \mathrm{H}, \mathrm{m}), 2.06-2.16(1 \mathrm{H}$, m), $2.39(1 \mathrm{H}, \mathrm{dd}, J=6.2,18.2 \mathrm{~Hz}), 3.48-3.56(1 \mathrm{H}, \mathrm{m}), 3.56-3.66(2 \mathrm{H}, \mathrm{m}), 6.39(1 \mathrm{H}, \mathrm{s}), 9.39(1 \mathrm{H}, \mathrm{s}) ;{ }^{13} \mathrm{C} \mathrm{NMR}(125 \mathrm{MHz}$, $\left.\mathrm{CDCl}_{3}\right) \delta$-5.2, -5.3, 5.4 (3 carbons), 7.2 (3 carbons), 17.0, 18.4, 18.5, 20.9, 23.1, 25.9, 26.0 (3 carbons), 26.7, 31.5, 35.0, 36.6, 40.0, 43.9, 64.2, 73.4, 137.6, 162.8, 195.1; FT-IR (neat) 2955, 2880, 2712, 1690, 1644, 1462, 1385, 1304, 1254, 1182, $1101,1043,1007,965,936,837,799,775,739,567,426 \mathrm{~cm}^{-1}$; HREIMS $(\mathrm{m} / z)$ calcd for $\mathrm{C}_{28} \mathrm{H}_{54} \mathrm{O}_{3} \mathrm{Si}_{2}\left(\mathrm{M}^{+}\right), 494.3611$, found 494.3611.

(4a $R, 5 S, 6 R, 8 \mathrm{a} R)$-5-(3-tert-Butyldimethylsilyloxypropyl)-5,8a-dimethyl-6-triethylsilyloxy-3,4,4a,5,6,7,8,8a-octahydronaphthalene-2-methanol (17).

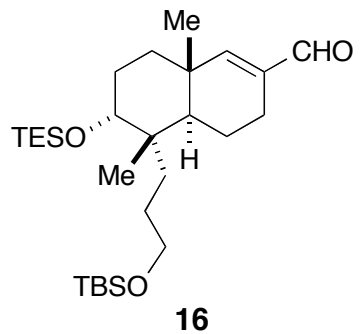

16

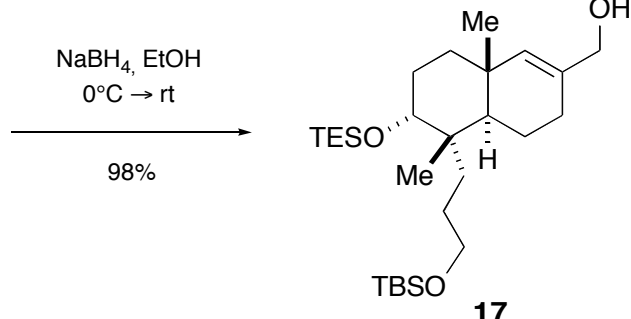

17 
Sodium borohydride $(34.0 \mathrm{mg}, 0.89 \mathrm{mmol})$ was added in small portions to a stirred solution of 16 (313 $\mathrm{mg}, 0.63 \mathrm{mmol}) \mathrm{in}$ ethyl alcohol $(13 \mathrm{ml})$ at $0^{\circ} \mathrm{C}$, and stirring was continued for $30 \mathrm{~min}$ at room temperature. The reaction was quenched with $10 \%$ aqueous acetic acid $(4 \mathrm{ml})$ at $0^{\circ} \mathrm{C}$, and the resulting mixture was extracted with ethyl acetate $(3 \times 35 \mathrm{ml})$. The combined extracts were washed with saturated aqueous sodium hydrogen carbonate $(2 \times 20 \mathrm{ml})$ and brine $(2 \times 20 \mathrm{ml})$, then dried over $\mathrm{Na}_{2} \mathrm{SO}_{4}$. Concentration of the solvent in vacuo afforded a residue, which was purified by column chromatography (hexaneethyl acetate, $10: 1)$ to give $17(311 \mathrm{mg}, 98 \%)$ as a colorless viscous liquid. $[\alpha]_{\mathrm{D}}{ }^{20}-5.3^{\circ}\left(c 1.19, \mathrm{CHCl}_{3}\right) ;{ }^{1} \mathrm{H} \mathrm{NMR}(500 \mathrm{MHz}$, $\left.\mathrm{CDCl}_{3}\right) \delta 0.05(6 \mathrm{H}, \mathrm{s}), 0.59-0.64(6 \mathrm{H}, \mathrm{m}), 0.79(3 \mathrm{H}, \mathrm{s}), 0.90(9 \mathrm{H}, \mathrm{s}), 0.93-0.98(9 \mathrm{H}, \mathrm{m}), 0.95(3 \mathrm{H}, \mathrm{s}), 1.14(1 \mathrm{H}, \mathrm{dt}, J=3.2$, $12.5 \mathrm{~Hz}), 1.22(1 \mathrm{H}, \mathrm{t}, J=6.0 \mathrm{~Hz}), 1.25-1.30(2 \mathrm{H}, \mathrm{m}), 1.34-1.45(1 \mathrm{H}, \mathrm{m}), 1.46-1.54(3 \mathrm{H}, \mathrm{m}), 1.61(1 \mathrm{H}, \mathrm{dd}, J=1.5,12.7 \mathrm{~Hz})$, 1.64-1.72 (2H, m), 1.82-1.92 (1H, m), 1.99-2.14 (2H, m), 3.48-3.55 (1H, m), 3.56-3.63 (2H, m), $3.96(2 \mathrm{H}, \mathrm{brd}, J=6.0 \mathrm{~Hz})$, $5.34(1 \mathrm{H}, \mathrm{s}) ;{ }^{13} \mathrm{C}$ NMR $\left(125 \mathrm{MHz}, \mathrm{CDCl}_{3}\right) \delta-5.2,-5.3,5.4$ (3 carbons), 7.2 (3 carbons), 17.9, 18.4, 18.5, $21.9,26.0$ (3 carbons), 26.3, 26.8, 27.4, 32.6, 34.9, 35.0, 39.7, 44.3, 64.4, 67.3, 73.8, 133.3, 136.9; IR (neat) 3328, 2955, 2280, 1462, 1414, $1385,1360,1254,1186,1101,1041,1007,965,837,775,739,681 \mathrm{~cm}^{-1}$; HREIMS (m/z) calcd for $\mathrm{C}_{28} \mathrm{H}_{56} \mathrm{O}_{3} \mathrm{Si}_{2}\left(\mathrm{M}^{+}\right)$, 496.3768 , found 496.3763 .

(4aR,5S,6R,8aR)-5-(3-tert-Butyldimethylsilyloxypropyl)-5,8a-dimethyl-2-tri- $n$-butylstannylmethoxymethyl-6-triethylsilyloxy-3,4,4a,5,6,7,8,8a-octahydronaphthalene (5).

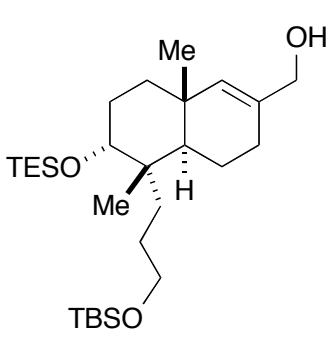

17

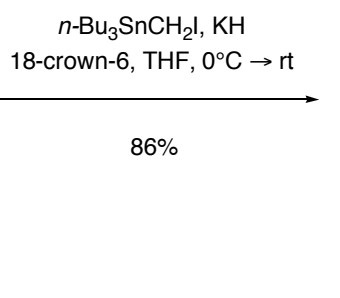

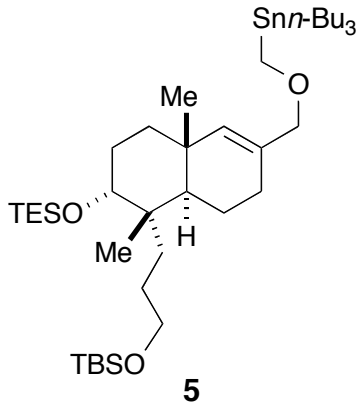

Potassium hydride (68.2 mg, $1.7 \mathrm{mmol}), 18$-crown-6 (450 mg, $1.7 \mathrm{mmol})$, and iodomethyl tri- $n$-butyltin (0.49 ml, 1.1 $\mathrm{mmol})$ were added successively to a stirred solution of $17(282 \mathrm{mg}, 0.57 \mathrm{mmol})$ in dry $\mathrm{THF}(8 \mathrm{ml})$ at $0^{\circ} \mathrm{C}$ under argon, and stirring was continued for $3 \mathrm{~h}$ at room temperature. The reaction was quenched with saturated aqueous ammonium chloride (4 $\mathrm{ml})$ at $0^{\circ} \mathrm{C}$, and the resulting mixture was extracted with ethyl acetate $(3 \mathrm{x} 40 \mathrm{ml})$. The combined extracts were washed with brine $(2 \times 30 \mathrm{ml})$, then dried over $\mathrm{Na}_{2} \mathrm{SO}_{4}$. Concentration of the solvent in vacuo afforded a residue, which was purified by column chromatography (hexane-ethyl acetate, $100: 1 \rightarrow 50: 1)$ to give $\mathbf{5}(391 \mathrm{mg}, 86 \%)$ as a colorless viscous liquid. $[\alpha]_{\mathrm{D}}{ }^{20}-$ $3.6^{\circ}\left(c 1.31, \mathrm{CHCl}_{3}\right) ;{ }^{1} \mathrm{H}$ NMR $\left(500 \mathrm{MHz}, \mathrm{CDCl}_{3}\right) \delta 0.04(6 \mathrm{H}, \mathrm{s}), 0.56-0.64(6 \mathrm{H}, \mathrm{m}), 0.78(3 \mathrm{H}, \mathrm{s}), 0.86-0.91(21 \mathrm{H}, \mathrm{m}), 0.93-$ $0.99(13 \mathrm{H}, \mathrm{m}), 1.13(1 \mathrm{H}, \mathrm{dt}, J=3.2,12.5 \mathrm{~Hz}), 1.24-1.35(9 \mathrm{H}, \mathrm{m}), 1.45-1.56(11 \mathrm{H}, \mathrm{m}), 1.59-1.74(3 \mathrm{H}, \mathrm{m}), 1.81-1.90(1 \mathrm{H}, \mathrm{m})$, 1.91-2.01 (1H, m), 2.02-2.11 (1H, m), 3.48-3.55 (1H, m), 3.55-3.62 (2H, m), 3.62-3.70 (4H, m), 5.30 (1H, s); ${ }^{13} \mathrm{C} \mathrm{NMR}$ (125MHz, $\left.\mathrm{CDCl}_{3}\right) \delta-5.2,-5.3,5.4$ (3 carbons), 7.2 (3 carbons), 9.0 (3 carbons), 13.7 (3 carbons), 17.9, 18.4, 18.5, 22.0, 26.0 (3 carbons), 26.3, 26.7, 27.3 (3 carbons), 27.6, 29.2 (3 carbons), 32.7, 34.9, 35.0, 39.7, 44.3, 60.4, 64.4, 73.8, 79.3, 130.7, 138.7; IR (neat) 2955, 1462, 1416, 1383, 1254, 1101, 1007, 961, 837, 775, 739, 683, 596, $511 \mathrm{~cm}^{-1}$; EIMS m/z: 743 [(M-n$\left.\mathrm{Bu})^{+}\right]$. 


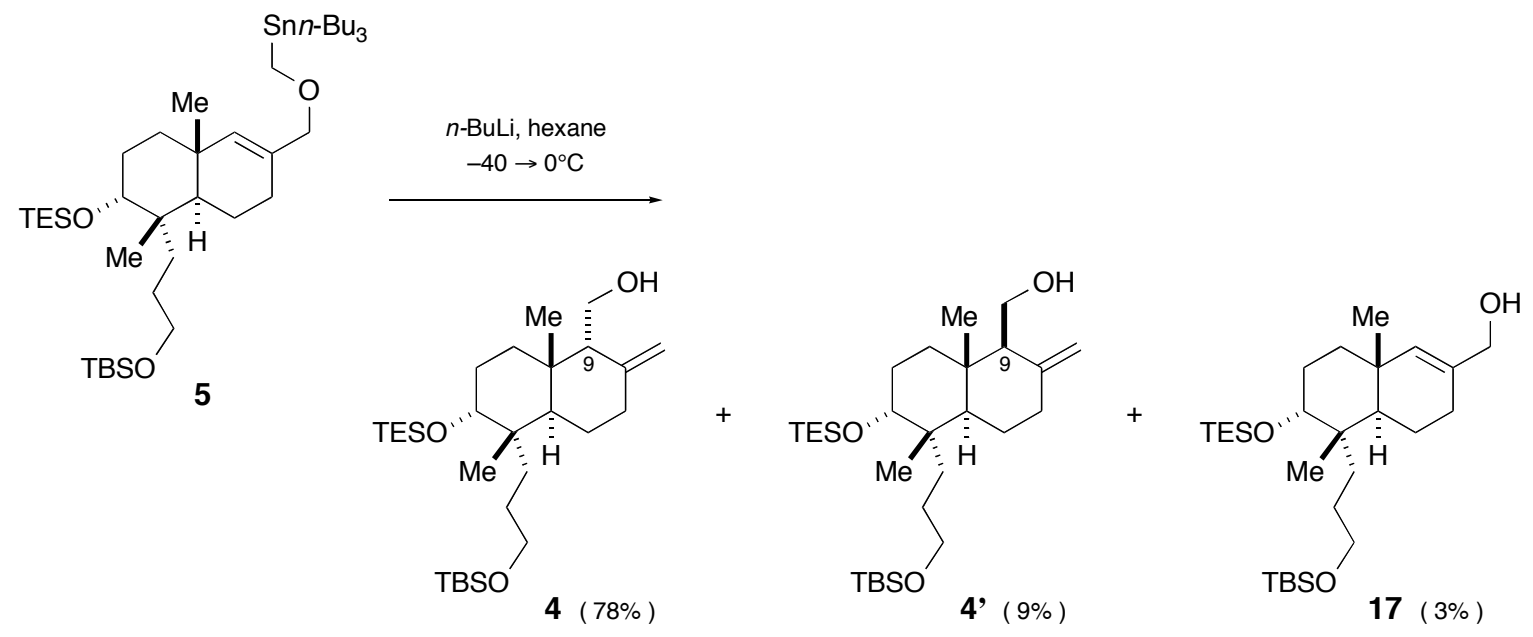

n-Butyllithium in hexane (1.58 M solution, $0.91 \mathrm{ml}, 1.4 \mathrm{mmol})$ was added dropwise to a stirred solution of 5 (116 mg, 0.14 $\mathrm{mmol})$ in dry degassed hexane $(3 \mathrm{ml})$ at $-40^{\circ} \mathrm{C}$ under argon, and the mixture was gradually warmed up to $0^{\circ} \mathrm{C}$ over $4 \mathrm{~h} . n$ Butyllithium in hexane $(1.58 \mathrm{M}$ solution, $0.91 \mathrm{ml}, 1.4 \mathrm{mmol})$ was added again to the mixture at $-40^{\circ} \mathrm{C}$, and stirring was continued for $16 \mathrm{~h}$ at $0{ }^{\circ} \mathrm{C}$. The reaction was quenched with saturated aqueous ammonium chloride $(6 \mathrm{ml})$ at $0{ }^{\circ} \mathrm{C}$, and the resulting mixture was extracted with ethyl acetate $(3 \times 30 \mathrm{ml})$. The combined extracts were washed with brine $(2 \times 20 \mathrm{ml})$, then dried over $\mathrm{Na}_{2} \mathrm{SO}_{4}$. Concentration of the solvent in vacuo afforded a residue, which was purified by column chromatography (hexane-ethyl acetate, 20:1) to give 4 (57.7 mg, 78\%) as white solid, 4' (6.6 mg, 9\%) as a colorless viscous liquid, and 17 (2.2 mg, 3\%). The stereostructures of 4 and 4' were confirmed by NOESY experiments in the $500 \mathrm{MHz}{ }^{1} \mathrm{H}$ NMR spectra. The key NOESY correlation is depicted in the following figure.

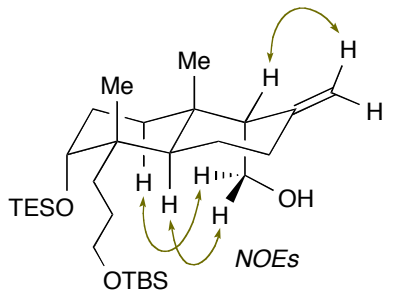

4

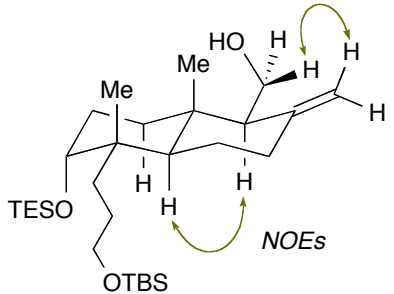

4'

$4\left(\mathrm{C} 9-\alpha-\mathrm{CH}_{2} \mathrm{OH}\right)$ : Recrystallization from hexane afforded white powder, mp $49-51{ }^{\circ} \mathrm{C} ;[\alpha]_{\mathrm{D}}{ }^{20}-19.3^{\circ}\left(c 1.20, \mathrm{CHCl}_{3}\right) ;{ }^{1} \mathrm{H}$ NMR (500MHz, CDCl $) \delta 0.04(6 \mathrm{H}, \mathrm{s}), 0.58-0.65(6 \mathrm{H}, \mathrm{m}), 0.76(3 \mathrm{H}, \mathrm{s}), 0.89(9 \mathrm{H}, \mathrm{s}), 0.97(9 \mathrm{H}, \mathrm{t}, J=7.9 \mathrm{~Hz}), 0.98(3 \mathrm{H}, \mathrm{s})$, 1.21-1.27 (3H, m), 1.27-1.39 (1H, m), 1.44-1.54 (4H, m), 1.56-1.64 (1H, m), $1.71(1 \mathrm{H}, \mathrm{dd}, J=2.8,12.9 \mathrm{~Hz}), 1.81-1.91(2 \mathrm{H}$, m), 1.95-2.04 (1H, m), 2.10-2.20 (1H, m), 2.29 (1H, br d, J=13.9 Hz), 3.47-3.53 (1H, m), 3.55-3.61 (2H, m), 3.62-3.69 (1H, $\mathrm{m}), 3.81(1 \mathrm{H}, \mathrm{dt}, J=4.9,10.0 \mathrm{~Hz}), 4.74(1 \mathrm{H}, \mathrm{t}, J=2.1 \mathrm{~Hz}), 4.91(1 \mathrm{H}, \mathrm{t}, J=2.1 \mathrm{~Hz}) ;{ }^{13} \mathrm{C} \mathrm{NMR}\left(125 \mathrm{MHz}, \mathrm{CDCl}_{3}\right) \delta-5.2,-5.3$, 5.4 (3 carbons), 7.2 (3 carbons), 18.4, 19.1, 22.4, 23.2, 26.0 (3 carbons), 26.1, 26.8, 29.0, 31.6, 35.4, 37.4, 40.0, 40.5, 59.0, 60.9, 64.3, 73.6, 112.7, 147.3; IR (KBr) 3341, 2957, 2880, 2361, 1653, 1462, 1414, 1385, 1360, 1256, 1101, 1034, 1011, 970, 936, 889, 837, 814, 777, 737, $666 \mathrm{~cm}^{-1}$; HREIMS $(\mathrm{m} / \mathrm{z})$ calcd for $\mathrm{C}_{29} \mathrm{H}_{58} \mathrm{O}_{3} \mathrm{Si}_{2}\left(\mathrm{M}^{+}\right), 510.3925$, found 510.3905 . 
4' $\left(\mathrm{C} 9-\beta-\mathrm{CH}_{2} \mathrm{OH}\right)$ : colorless viscous liquid. $[\alpha]_{\mathrm{D}}{ }^{20}-17.3^{\circ}\left(c 0.83, \mathrm{CHCl}_{3}\right) ;{ }^{1} \mathrm{H} \mathrm{NMR}\left(500 \mathrm{MHz}, \mathrm{CDCl}_{3}\right) \delta 0.04(6 \mathrm{H}, \mathrm{s})$, $0.58-0.61(6 \mathrm{H}, \mathrm{m}), 0.74(3 \mathrm{H}, \mathrm{s}), 0.75(3 \mathrm{H}, \mathrm{s}), 0.89(9 \mathrm{H}, \mathrm{s}), 0.98(9 \mathrm{H}, \mathrm{t}, J=7.9 \mathrm{~Hz}), 1.21-1.35(4 \mathrm{H}, \mathrm{m}), 1.36-1.41(1 \mathrm{H}, \mathrm{m})$, 1.45-1.55 (4H, m), 1.60-1.71 (2H, m), 1.73-1.83 (1H, m), 2.01-2.10 (2H, m), 2.38-2.46 (1H, m), 3.47-3.54 (1H, m), 3.55$3.61(2 \mathrm{H}, \mathrm{m}), 3.73-3.80(1 \mathrm{H}, \mathrm{m}), 3.81-3.88(1 \mathrm{H}, \mathrm{m}), 4.64(1 \mathrm{H}, \mathrm{d}, J=1.0 \mathrm{~Hz}), 4.93(1 \mathrm{H}, \mathrm{d}, J=1.0 \mathrm{~Hz}) ;{ }^{13} \mathrm{C} \mathrm{NMR}(125 \mathrm{MHz}$, $\left.\mathrm{CDCl}_{3}\right) \delta-5.2,-5.3,5.4$ (3 carbons), 7.2 (3 carbons), 16.0, 18.4, 19.0, 23.5, 26.0 (3 carbons), 26.3, 26.8, 31.5, 35.6, 37.8, 38.8, 40.1, 48.2, 58.8, 59.2, 64.3, 73.3, 106.1, 148.0; IR (neat) 3404, 2953, 2878, 1644, 1462, 1385, 1362, 1254, 1100, 1053, $1009,965,937,891,837,814,775,739 \mathrm{~cm}^{-1}$; HREIMS $(\mathrm{m} / z)$ calcd for $\mathrm{C}_{29} \mathrm{H}_{58} \mathrm{O}_{3} \mathrm{Si}_{2}\left(\mathrm{M}^{+}\right), 510.3925$, found 510.3938 .

$(1 R, 4 a R, 5 S, 6 R, 8 \mathrm{a} S)-5$-(3-tert-Butyldimethylsilyloxypropyl)-5,8a-dimethyl-2-methylene-6-(triethylsilyloxy)decahydronaphthalene-1-carbaldehyde (3).
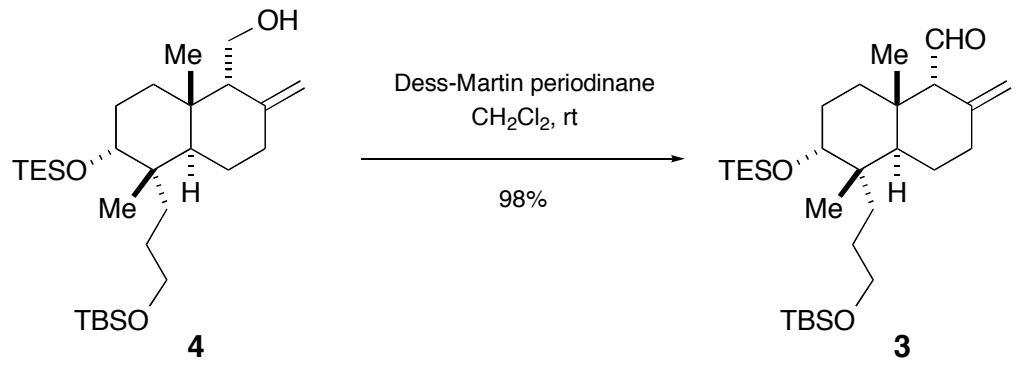

Dess-Martin periodinane (366 mg, $0.86 \mathrm{mmol})$ was added in small portions to a stirred solution of 4 (147 $\mathrm{mg}, 0.29 \mathrm{mmol})$ in dry dichloromethane $(12 \mathrm{ml})$ at room temperature. After $1 \mathrm{~h}$, the reaction was quenched with $20 \%$ aqueous sodium thiosulfate $(10 \mathrm{ml})$ at $0^{\circ} \mathrm{C}$, and the resulting mixture was extracted with diethyl ether $(2 \times 50 \mathrm{ml})$. The combined extracts were washed successively with $10 \%$ aqueous sodium thiosulfate $(2 \times 20 \mathrm{ml})$, saturated aqueous sodium hydrogen carbonate $(2 \times 20$ $\mathrm{ml})$, and brine $(2 \times 20 \mathrm{ml})$, then dried over $\mathrm{Na}_{2} \mathrm{SO}_{4}$. Concentration of the solvent in vacuo afforded a residue, which was purified by column chromatography (hexane-ethyl acetate, $50: 1)$ to give $\mathbf{3}(143 \mathrm{mg}, 98 \% \text { ) as a colorless viscous liquid. [ } \alpha]_{\mathrm{D}}{ }^{20}$ $-3.1^{\circ}$ ( c 1.22, $\left.\mathrm{CHCl}_{3}\right) ;{ }^{1} \mathrm{H} \mathrm{NMR}\left(500 \mathrm{MHz}, \mathrm{CDCl}_{3}\right) \delta 0.05(6 \mathrm{H}, \mathrm{s}), 0.57-0.64(6 \mathrm{H}, \mathrm{m}), 0.80(3 \mathrm{H}, \mathrm{s}), 0.90(9 \mathrm{H}, \mathrm{s}), 0.96(9 \mathrm{H}, \mathrm{t}$, $J=7.9 \mathrm{~Hz}), 0.98(3 \mathrm{H}, \mathrm{s}), 1.09(1 \mathrm{H}, \mathrm{dt}, J=3.1,12.9 \mathrm{~Hz}), 1.24-1.35(2 \mathrm{H}, \mathrm{m}), 1.37-1.54(4 \mathrm{H}, \mathrm{m}), 1.69-1.77(1 \mathrm{H}, \mathrm{m}), 1.83-1.93$ $(1 \mathrm{H}, \mathrm{m}), 1.99-2.11(2 \mathrm{H}, \mathrm{m}), 2.26-2.36(1 \mathrm{H}, \mathrm{m}), 2.41-2.47(1 \mathrm{H}, \mathrm{m}), 2.49(1 \mathrm{H}, \mathrm{d}, J=3.9 \mathrm{~Hz}), 3.48-3.55(1 \mathrm{H}, \mathrm{m}), 3.56-3.66$ $(2 \mathrm{H}, \mathrm{m}), 4.73(1 \mathrm{H}, \mathrm{s}), 4.89(1 \mathrm{H}, \mathrm{s}), 10.03(1 \mathrm{H}, \mathrm{d}, J=3.9 \mathrm{~Hz}) ;{ }^{13} \mathrm{C}$ NMR $\left(125 \mathrm{MHz}, \mathrm{CDCl}_{3}\right) \delta-5.2,-5.3,5.4$ (3 carbons), $7.1(3$ carbons), 18.4, 18.9, 22.2, 22.3, 25.8, 26.0 (3 carbons), 26.7, 30.1, 33.7, 35.3, 38.9, 40.1, 42.2, 64.2, 71.4, 73.3, 113.1, 143.1, 203.4; IR (neat) 2955, 2880, 2730, 1719, 1647, 1462, 1414, 1385, 1362, 1258, 1100, 1013, 966, 895, 837, 810, 777, 739 cm ; HREIMS $(m / z)$ calcd for $\mathrm{C}_{29} \mathrm{H}_{56} \mathrm{O}_{3} \mathrm{Si}_{2}\left(\mathrm{M}^{+}\right), 508.3768$, found 508.3750.

3-Bromo-2-methoxy-5,6-dimethyl-4H-pyran-4-one (ii) and 3-bromo-4-methoxy-5,6-dimethyl-2H-pyran-2-one (iii)<smiles>Cc1oc(=O)cc(O)c1C</smiles>

i
1) $\mathrm{N}$-bromosuccinimide $\mathrm{Et}_{3} \mathrm{~N}, \mathrm{THF}, 0^{\circ} \mathrm{C}$

2) $\mathrm{CH}_{2} \mathrm{~N}_{2}, \mathrm{MeOH}, 0^{\circ} \mathrm{C}$<smiles>COc1oc([N+](=O)[O-])c(C)c(=O)c1Br</smiles>

ii ( $43 \%, 2$ steps )<smiles>COc1c([Na])oc(=O)c(Br)c1[N+](=O)[O-]</smiles>

iii ( $42 \%, 2$ steps ) 
$\mathrm{N}$-Bromosuccsinimide $(524 \mathrm{mg}, 3.0 \mathrm{mmol})$ was added in small portions to a stirred solution of 4-hydroxy-5,6-dimethyl$2 H$-pyran-2-one (i) $(413 \mathrm{mg}, 3.0 \mathrm{mmol})$ in dry THF $(16 \mathrm{ml})$ containing triethylamine $(0.41 \mathrm{ml}, 3.0 \mathrm{mmol})$ at $0^{\circ} \mathrm{C}$. After $1 \mathrm{~h}$, the reaction mixture was concentrated in vacuo to afford 3-bromo-4-hydroxy-5,6-dimethyl- 2H-pyran-2-one (960 mg) as a brown oil, which was used for the next reaction without further purification.

Diazomethane in diethyl ether $(1.0 \mathrm{M}$ solution, $3.30 \mathrm{ml}, 3.3 \mathrm{mmol})$ was added dropwise to a stirred suspension of the crude brominated product $(960 \mathrm{mg})$ in methanol $(20 \mathrm{ml})$ at $0^{\circ} \mathrm{C}$. After $1 \mathrm{~h}$, the reaction was quenched with acetic acid $(0.8 \mathrm{ml})$ at $0^{\circ} \mathrm{C}$. The resulting mixture was extracted with ethyl acetate $(3 \times 60 \mathrm{ml})$, and the combined extracts were washed with saturated aqueous sodium hydrogen carbonate $(2 \times 60 \mathrm{ml})$ and brine $(2 \times 60 \mathrm{ml})$, then dried over $\mathrm{MgSO}_{4}$. Concentration of the solvent in vacuo afforded a residue, which was purified by column chromatography (hexane-ethyl acetate, 1:1) to give ii (295 $\mathrm{mg}, 43 \%, 2$ steps) as a more polar product and iii (288 mg, 42\%, 2 steps) as a less polar product.

ii ( $\gamma$-pyrone) : white needles (recrystallization from acetone); mp $110-111^{\circ} \mathrm{C} ;{ }^{1} \mathrm{H} \mathrm{NMR}(500 \mathrm{MHz}, \mathrm{CDCl} 3) \delta 1.99(3 \mathrm{H}, \mathrm{s})$, $2.31(3 \mathrm{H}, \mathrm{s}), 4.06(3 \mathrm{H}, \mathrm{s}) ;{ }^{13} \mathrm{C}$ NMR $\left(125 \mathrm{MHz}, \mathrm{CDCl}_{3}\right) \delta 10.7,16.8,56.3,89.2,118.8,155.1,161.8,175.3 ; \mathrm{IR}(\mathrm{KBr}) 2961$, 2930, 2866, 1665, 1626, 1586, 1460, 1393, 1368, 1327, 1217, 1013, 1179, 1138, 1065, 990, 955, 924, 804, 752, 664, 608, 586, $469 \mathrm{~cm}^{-1}$; Anal calcd for $\mathrm{C}_{8} \mathrm{H}_{9} \mathrm{BrO}_{3}$ : C, 41.23; H, 3.89, found C, 41.32; H, 3.95.

iii ( $\alpha$-pyrone) : white needles (recrystallization from acetone); mp $78-79^{\circ} \mathrm{C} ;{ }^{1} \mathrm{H} \mathrm{NMR}\left(500 \mathrm{MHz}, \mathrm{CDCl}_{3}\right) \delta 1.96(3 \mathrm{H}, \mathrm{s})$, $2.23(3 \mathrm{H}, \mathrm{s}), 4.06(3 \mathrm{H}, \mathrm{s}) ;{ }^{13} \mathrm{C}$ NMR $\left(125 \mathrm{MHz}, \mathrm{CDCl}_{3}\right) \delta 10.7,17.4,60.7,95.6,110.2,157.2,161.2,169.3 ; \mathrm{IR}(\mathrm{KBr}) 2949$, 2857, 1890, 1740, 1642, 1535, 1447, 1400, 1356, 1277, 1215, 1179, 1136, 1113, 1022, 984, 949, 909, 808, 754, 721, 648, $544,459 \mathrm{~cm}^{-1}$; Anal calcd for $\mathrm{C}_{8} \mathrm{H}_{9} \mathrm{BrO}_{3}: \mathrm{C}, 41.23 ; \mathrm{H}, 3.89$, found $\mathrm{C}, 41.00 ; \mathrm{H}, 4.02$.

\section{3-[(1R,4a $R, 5 S, 6 R, 8 \mathrm{a} S)$-5-(3-tert-Butyldimethylsilyloxypropyl)-5,8a-dimethyl-2-methylene-6-(triethylsilyloxy)deca-} hydronaphthalen-1-yl]hydroxymethyl-2-methoxy-5,6-dimethyl-4H-pyran-4-one (18).
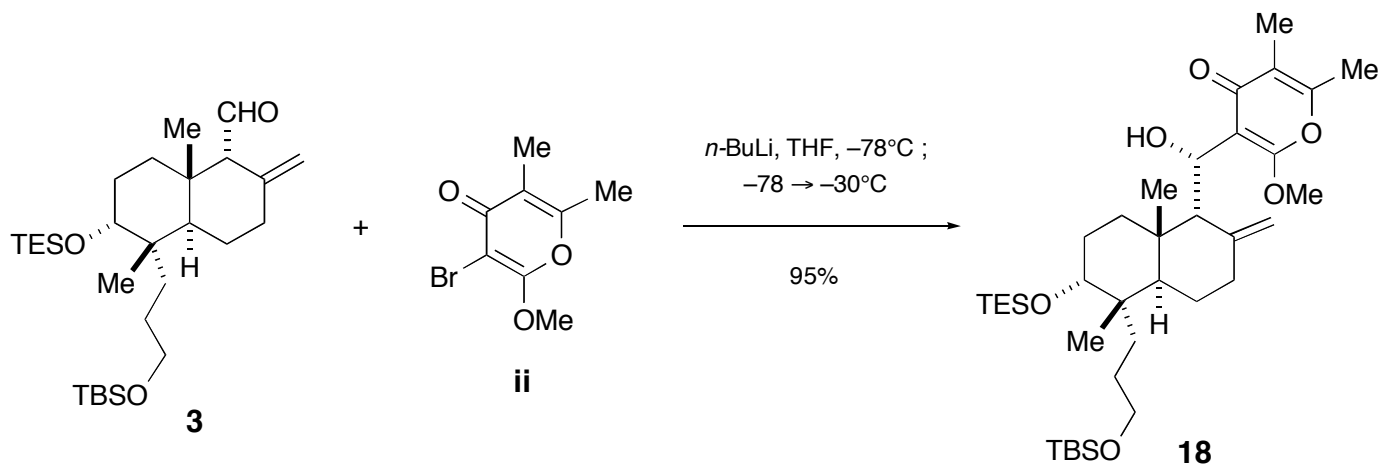

$n$-Butyllithium in hexane $(1.58 \mathrm{M}$ solution, $0.66 \mathrm{ml}, 1.0 \mathrm{mmol})$ was added dropwise to a stirred solution of 3-bromo-2methoxy-5,6-dimethyl-4H-pyran-4-one (ii) $(254 \mathrm{mg}, 1.1 \mathrm{mmol})$ in dry THF $(8 \mathrm{ml})$ at $-78^{\circ} \mathrm{C}$ under argon. After $30 \mathrm{~min}$, a solution of 3 (139 mg, $0.27 \mathrm{mmol})$ in dry THF $(8 \mathrm{ml})$ was added to the above mixture at $-78^{\circ} \mathrm{C}$, and stirring was continued for $2 \mathrm{~h}$ at $-30^{\circ} \mathrm{C}$. The reaction was quenched with saturated aqueous ammonium chloride $(8 \mathrm{ml})$ at $-30^{\circ} \mathrm{C}$, and the resulting mixture was extracted with diethyl ether $(3 \times 50 \mathrm{ml})$. The combined extracts were washed with brine $(3 \mathrm{x} 30 \mathrm{ml})$, then dried over $\mathrm{Na}_{2} \mathrm{SO}_{4}$. Concentration of the solvent in vacuo afforded a residue, which was purified by column chromatography (hexane-ethyl acetate, $5: 1)$ to give $\mathbf{1 8}(172 \mathrm{mg}, 95 \%)$ as a colorless oil. $[\alpha]_{\mathrm{D}}{ }^{20}-17.7^{\circ}\left(c 1.13, \mathrm{CHCl}_{3}\right) ;{ }^{1} \mathrm{H} \mathrm{NMR}(500 \mathrm{MHz}$, $\left.\mathrm{CDCl}_{3}\right) \delta 0.05(6 \mathrm{H}, \mathrm{s}), 0.58-0.69(6 \mathrm{H}, \mathrm{m}), 0.77(3 \mathrm{H}, \mathrm{s}), 0.80-0.87(1 \mathrm{H}, \mathrm{m}), 0.90(9 \mathrm{H}, \mathrm{s}), 0.94(3 \mathrm{H}, \mathrm{s}), 0.99(9 \mathrm{H}, \mathrm{t}, J=7.9 \mathrm{~Hz})$, 1.25-1.41 (3H, m), 1.48-1.55 (3H, m), 1.58-1.66 (1H, m), 1.82-1.92 (2H, m), $1.87(3 \mathrm{H}, \mathrm{s}), 2.25(3 \mathrm{H}, \mathrm{s}), 2.30-2.37$ (2H, m), $2.44(1 \mathrm{H}, \mathrm{dd}, J=2.9,12.8 \mathrm{~Hz}), 2.50-2.60(1 \mathrm{H}, \mathrm{m}), 3.48-3.54(1 \mathrm{H}, \mathrm{m}), 3.55-3.65(2 \mathrm{H}, \mathrm{m}), 3.95(3 \mathrm{H}, \mathrm{s}), 4.32(1 \mathrm{H}, \mathrm{s}), 4.72(1 \mathrm{H}$, 
s), $4.84(1 \mathrm{H}, \mathrm{d}, J=9.9 \mathrm{~Hz}), 5.22(1 \mathrm{H}, \mathrm{dd}, J=4.4,9.9 \mathrm{~Hz}) ;{ }^{13} \mathrm{C} \mathrm{NMR}\left(125 \mathrm{MHz}, \mathrm{CDCl}_{3}\right) \delta-5.2,-5.3,5.4$ (3 carbons), $7.2(3$ carbons), 9.7, 16.9, 18.4, 19.6, 23.0, 23.2, 26.0 (3 carbons), 26.5, 27.1, 28.9, 35.0, 35.6, 38.5, 40.2, 40.3, 55.7, 62.0, 64.5, 67.7, 74.2, 105.7, 111.6, 119.5, 148.7, 155.3, 161.0, 181.6; FT-IR (neat) 3301, 2955, 2878, 1671, 1588, 1464, 1424, 1379, 1319, 1296, 1254, 1152, 1096, 1009, 937, 835, 802, 779, 741, $669 \mathrm{~cm}^{-1}$; HREIMS $(\mathrm{m} / z)$ calcd for $\mathrm{C}_{37} \mathrm{H}_{66} \mathrm{O}_{6} \mathrm{Si}_{2}\left(\mathrm{M}^{+}\right), 662.4398$, found 662.4383 .

\section{3-[(1R,4a $R, 5 S, 6 R, 8 \mathrm{a} R)$-5-(3-tert-Butyldimethylsilyloxypropyl)-5,8a-dimethyl-2-methylene-6-(triethylsilyloxy)deca- hydronaphthalen-1-yl]methyl-2-methoxy-5,6-dimethyl-4H-pyran-4-one (20).}
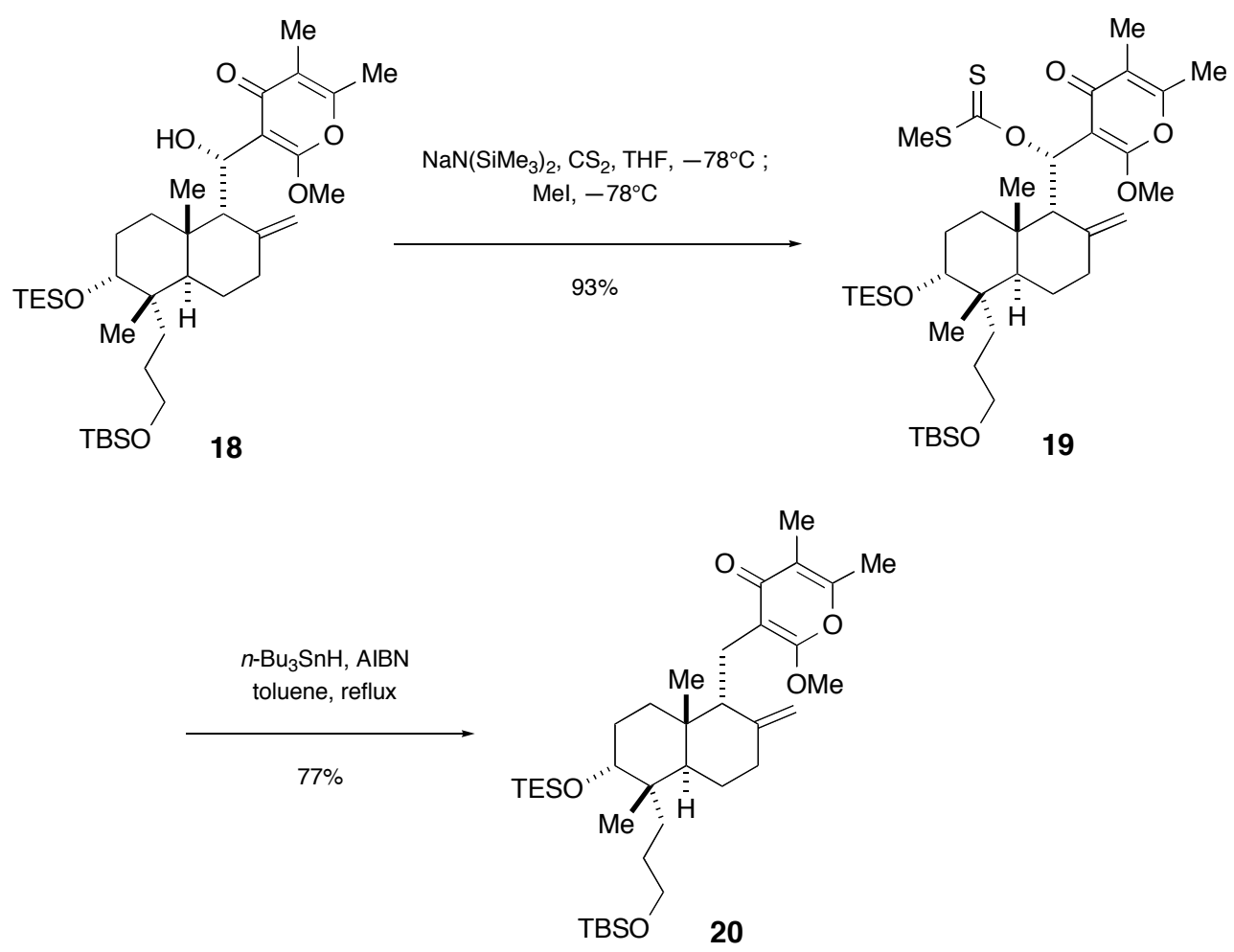

Sodium bis(trimethylsilyl)amide in THF (1.0 M solution, $0.312 \mathrm{ml}, 0.31 \mathrm{mmol})$ was added dropwise to a stirred solution of $18(172 \mathrm{mg}, 0.26 \mathrm{mmol})$ in dry $\mathrm{THF}(5 \mathrm{ml})$ containing carbon disulfide $(0.31 \mathrm{ml}, 5.2 \mathrm{mmol})$ at $-78^{\circ} \mathrm{C}$ under argon. After $1 \mathrm{~h}$, methyl iodide $(0.20 \mathrm{ml}, 2.6 \mathrm{mmol})$ was added dropwise to the mixture at $-78^{\circ} \mathrm{C}$, and the resulting solution was further stirred for $1 \mathrm{~h}$ at the same temperature. The reaction was quenched with saturated aqueous ammonium chloride (4 ml), and the resulting mixture was extracted with ethyl acetate $(3 \times 40 \mathrm{ml})$. The combined extracts were washed with brine $(3 \mathrm{x} 30 \mathrm{ml})$, then dried over $\mathrm{Na}_{2} \mathrm{SO}_{4}$. Concentration of the solvent in vacuo afforded a residue, which was purified by column chromatography (hexane-ethyl acetate, $5: 1)$ to give 3-[(1R,4a $R, 5 S, 6 R, 8 \mathrm{a} S)-5$-(3-tert-butyldimethylsilyloxypropyl)-5,8adimethyl-2-methylene-6-(triethylsilyloxy)decahydoronaphtalen-1-yl](methyldithiocarbonyloxy)methyl-2-methoxy-5,6dimethyl-4H-pyran-4-one (19) (182 mg, 93\%) as a pale yellow viscous liquid. HREIMS (m/z) calcd for $\mathrm{C}_{39} \mathrm{H}_{68} \mathrm{O}_{6} \mathrm{~S}_{2} \mathrm{Si}_{2}\left(\mathrm{M}^{+}\right)$, 752.3996 , found 752.3999 .

Tributyltin hydride $(0.132 \mathrm{ml}, 0.48 \mathrm{mmol})$ and 2,2'-azobisisobutyronitrile (AIBN) (7.9 $\mathrm{mg}, 48 \mu \mathrm{mol})$ were added successively to a stirred solution of $\mathbf{1 9}(182 \mathrm{mg}, 0.24 \mathrm{mmol})$ in dry toluene $(8 \mathrm{ml})$ at room temperature. For the deaeration of the reaction mixture, it was frozen using liquid nitrogen, and the reaction vessel was evacuated in vacuo for 30 min followed 
by filled with dry argon. The mixture was heated at reflux for $1 \mathrm{~h}$ under argon. After cooling, the reaction mixture was concentrated in vacuo to afford a residue, which was purified by column chromatography (hexane-ethyl acetate, 10:1) to give $20(120 \mathrm{mg}, 77 \%)$ as a colorless viscous liquid. $[\alpha]_{\mathrm{D}}{ }^{20}-43.7^{\circ}\left(c 0.41, \mathrm{CHCl}_{3}\right) ;{ }^{1} \mathrm{H} \mathrm{NMR}\left(500 \mathrm{MHz}, \mathrm{CDCl}_{3}\right) \delta 0.05(6 \mathrm{H}, \mathrm{s})$, 0.58-0.66 (6H, m), $0.77(3 \mathrm{H}, \mathrm{s}), 0.90(9 \mathrm{H}, \mathrm{s}), 0.94-1.00(1 \mathrm{H}, \mathrm{m}), 0.95(3 \mathrm{H}, \mathrm{s}), 0.97(9 \mathrm{H}, \mathrm{t}, J=7.9 \mathrm{~Hz}), 1.24-1.38(3 \mathrm{H}, \mathrm{m})$, 1.45-1.55 (4H, m), 1.84-1.95 (3H, m), $1.90(3 \mathrm{H}, \mathrm{s}), 2.07-2.18(1 \mathrm{H}, \mathrm{m}), 2.23(3 \mathrm{H}, \mathrm{s}), 2.28-2.43(2 \mathrm{H}, \mathrm{m}), 2.48(1 \mathrm{H}, \mathrm{t}, J=12.8$ $\mathrm{Hz}), 2.75(1 \mathrm{H}, \mathrm{dd}, J=3.4,12.8 \mathrm{~Hz}), 3.45-3.55(1 \mathrm{H}, \mathrm{m}), 3.56-3.63(2 \mathrm{H}, \mathrm{m}), 3.84(3 \mathrm{H}, \mathrm{s}), 4.14(1 \mathrm{H}, \mathrm{t}, J=2.4 \mathrm{~Hz}), 4.47(1 \mathrm{H}, \mathrm{t}$, $J=2.4 \mathrm{~Hz}$ ); ${ }^{13} \mathrm{C}$ NMR (125 MHz, $\mathrm{CDCl}_{3}$ ) $\delta-5.2,-5.3,5.5$ (3 carbons), 7.2 (3 carbons), 10.0, 17.0, 18.4, 19.1, 19.8, 22.9, 23.1, 26.0 (3 carbons), 26.2, 26.9, 28.6, 31.5, 35.4, 37.8, 39.2, 39.9, 55.3, 56.3, 64.5, 74.0, 103.8, 108.8, 118.6, 124.9, 149.6, 154.8, 180.4; IR (neat); 2955, 2282, 1736, 1672, 1605, 1460, 1414, 1375, 1318, 1256, 1163, 1100, 1009, 835, 810, 779, 725 cm HREIMS $(m / z)$ calcd for $\mathrm{C}_{37} \mathrm{H}_{66} \mathrm{O}_{5} \mathrm{Si}_{2}\left(\mathrm{M}^{+}\right), 646.4449$, found 646.4454 .

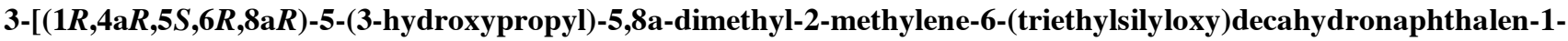 yl]methyl-2-methoxy-5,6-dimethyl-4H-pyran-4-one (21).}
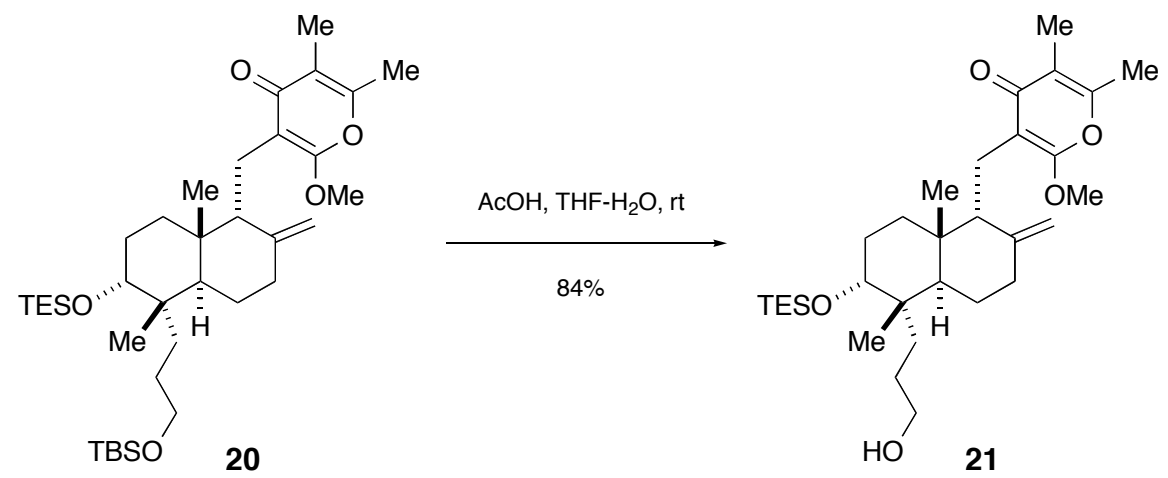

A solution of $20(94.0 \mathrm{mg}, 0.15 \mathrm{mmol})$ in acetic acid-THF- $\mathrm{H}_{2} \mathrm{O}(3: 2: 2)(16 \mathrm{ml})$ was stirred for $2 \mathrm{~h}$ at room temperature. The reaction mixture was basified with $1 \mathrm{M}$ sodium hydroxide $(6 \mathrm{ml})$ and saturated aqueous sodium hydrogen carbonate (10 ml) at $0^{\circ} \mathrm{C}$. The resulting mixture was extracted with ethyl acetate $(3 \times 50 \mathrm{ml})$. The combined extracts were washed with saturated aqueous ammonium chloride $(2 \times 30 \mathrm{ml})$ and brine $(2 \times 30 \mathrm{ml})$, and dried over $\mathrm{Na}_{2} \mathrm{SO}_{4}$. Concentration of the solvent in vacuo afforded a residue, which was purified by column chromatography (hexane-ethyl acetate, 2:1) to give 21 (64.9 $\mathrm{mg}, 84 \%)$ as a colorless viscous liquid. $[\alpha]_{\mathrm{D}}{ }^{20}-45.6^{\circ}\left(c 0.80, \mathrm{CHCl}_{3}\right) ;{ }^{1} \mathrm{H}$ NMR $\left(500 \mathrm{MHz}, \mathrm{CDCl}_{3}\right) \delta 0.59-0.67(6 \mathrm{H}, \mathrm{m}), 0.78(3 \mathrm{H}, \mathrm{s}), 0.94-$ $1.01(1 \mathrm{H}, \mathrm{m}), 0.95(3 \mathrm{H}, \mathrm{s}), 0.98(9 \mathrm{H}, \mathrm{t}, J=7.9 \mathrm{~Hz}), 1.15-1.22(1 \mathrm{H}, \mathrm{m}), 1.24-1.36(3 \mathrm{H}, \mathrm{m}), 1.40-1.48(1 \mathrm{H}, \mathrm{m}), 1.54-1.62(3 \mathrm{H}$, m), 1.83-1.94 (1H, m), $1.90(3 \mathrm{H}, \mathrm{s}), 2.07-2.14(1 \mathrm{H}, \mathrm{m}), 2.23(3 \mathrm{H}, \mathrm{s}), 2.29-2.44(2 \mathrm{H}, \mathrm{m}), 2.48(1 \mathrm{H}, \mathrm{t}, J=12.8 \mathrm{~Hz}), 2.75(1 \mathrm{H}$, dd, $J=3.5,12.8 \mathrm{~Hz}), 3.55-3.68(3 \mathrm{H}, \mathrm{m}), 3.85(3 \mathrm{H}, \mathrm{s}), 4.14(1 \mathrm{H}, \mathrm{t}, J=2.4 \mathrm{~Hz}), 4.48(1 \mathrm{H}, \mathrm{t}, J=2.4 \mathrm{~Hz}) ;{ }^{13} \mathrm{C} \mathrm{NMR}(125 \mathrm{MHz}$, $\mathrm{CDCl}_{3}$ ): $\delta 5.5$ (3 carbons), 7.2 (3 carbons), 10.0, 17.0, 19.2, 19.7, 22.9, 23.1, 26.2, 26.8, 28.6, 31.5, 35.4, 37.8, 39.2, 39.9, 55.3, 56.2, 64.3, 74.0, 103.7, 108.9, 118.6, 149.4, 154.8, 163.0, 180.4; FT-IR (neat) 3409, 3065, 2955, 2878, 1726, 1671, $1589,1462,1420,1377,1319,1258,1182,1148,1078,1011,885,810,750,478 \mathrm{~cm}^{-1}$; HREIMS $(\mathrm{m} / z)$ calcd for $\mathrm{C}_{31} \mathrm{H}_{52} \mathrm{O}_{5} \mathrm{Si}$ $\left(\mathrm{M}^{+}\right), 532.3584$, found 532.3555. 
3-[(1R,4a $R, 5 S, 6 R, 8 \mathrm{a} R)$-5,8a-dimethyl-2-methylene-5-(3-oxopropyl)-6-(triethylsilyloxy)decahydronaphthalen-1-yl]methyl-2-methoxy-5,6-dimethyl-4H-pyran-4-one (22).
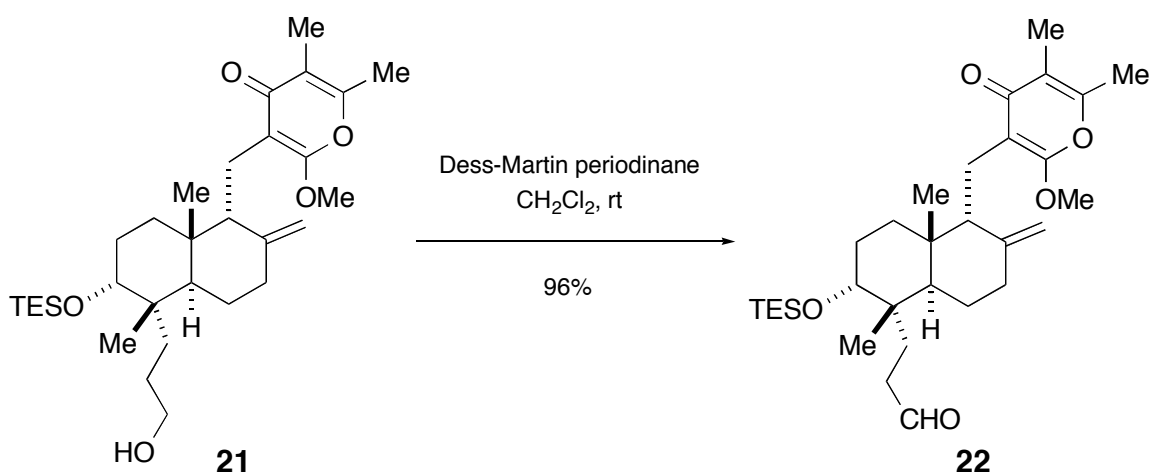

Dess-Martin periodinane (134 mg, $0.30 \mathrm{mmol})$ was added in small portions to a stirred solution of 21 (56.0 $\mathrm{mg}, 0.10 \mathrm{mmol})$ in dry dichloromethane $(6 \mathrm{ml})$ at room temperature. After $30 \mathrm{~min}$, the reaction was quenched with $20 \%$ aqueous sodium thiosulfate $(6 \mathrm{ml})$ at $0^{\circ} \mathrm{C}$, and the resulting mixture was extracted with diethyl ether $(3 \mathrm{x} 50 \mathrm{ml})$. The combined extracts were washed with saturated aqueous sodium hydrogen carbonate $(2 \times 30 \mathrm{ml})$ and brine $(2 \times 30 \mathrm{ml})$, then dried over $\mathrm{Na}_{2} \mathrm{SO}_{4}$. Concentration of the solvent in vacuo afforded a residue, which was purified by column chromatography (hexane-ethyl acetate, $4: 1)$ to give $22(53.5 \mathrm{mg}, 96 \%)$ as a colorless viscous liquid. [ $\alpha]_{\mathrm{D}}{ }^{20}-46.7^{\circ}\left(c 0.65, \mathrm{CHCl}_{3}\right) ;{ }^{1} \mathrm{H} \mathrm{NMR}(500 \mathrm{MHz}$, $\left.\mathrm{CDCl}_{3}\right) \delta 0.58-0.67(6 \mathrm{H}, \mathrm{m}), 0.78(3 \mathrm{H}, \mathrm{s}), 0.94-0.99(12 \mathrm{H}, \mathrm{m}), 0.99-1.05(1 \mathrm{H}, \mathrm{m}), 1.2-1.39(2 \mathrm{H}, \mathrm{m}), 1.55-1.66(2 \mathrm{H}, \mathrm{m})$, 1.69-1.78 (1H, m), 1.84-1.98 (3H, m), $1.91(3 \mathrm{H}, \mathrm{s}), 2.08-2.15(1 \mathrm{H}, \mathrm{m}), 2.24(3 \mathrm{H}, \mathrm{s}), 2.28-2.37(1 \mathrm{H}, \mathrm{m}), 2.38-2.51(4 \mathrm{H}, \mathrm{m})$, $2.76(1 \mathrm{H}, \mathrm{dd}, J=3.5,12.9 \mathrm{~Hz}), 3.58(1 \mathrm{H}, \mathrm{br} \mathrm{s}), 3.85(3 \mathrm{H}, \mathrm{s}), 4.15(1 \mathrm{H}, \mathrm{t}, J=2.3 \mathrm{~Hz}), 4.49(1 \mathrm{H}, \mathrm{t}, J=2.3 \mathrm{~Hz}), 9.80(1 \mathrm{H}, \mathrm{d}, J=1.6$ $\mathrm{Hz}) ;{ }^{13} \mathrm{C}$ NMR (125 MHz, $\mathrm{CDCl}_{3}$ ) $\delta 5.4$ (3 carbons), 7.2 (3 carbons), 10.0, 17.0, 19.4, 19.8, 23.0, 26.1, 28.5, 29.7, 31.4, 31.5, 37.7, 38.8, 39.1, 39.7, 55.3, 56.0, 74.3, 103.5, 109.2, 118.6, 149.1, 154.8, 163.0, 180.4, 203.6; IR (neat) 2953, 1726, 1672,

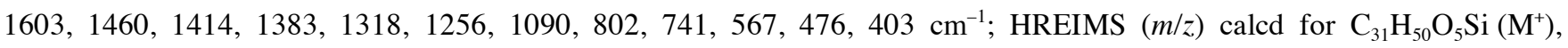
530.3428 , found 530.3447 .

3-[[(4a $R, 6 \mathrm{a} R, 7 R, 10 \mathrm{a} R, 10 \mathrm{~b} R)-3-H y d r o x y-6 a, 10 \mathrm{~b}-d i m e t h y l-8$-(methylene)dodecahydro-1H-benzo[f]chromen-7-yl]methyl]-2-methoxy-5,6-dimethyl-4H-pyran-4-one(23).
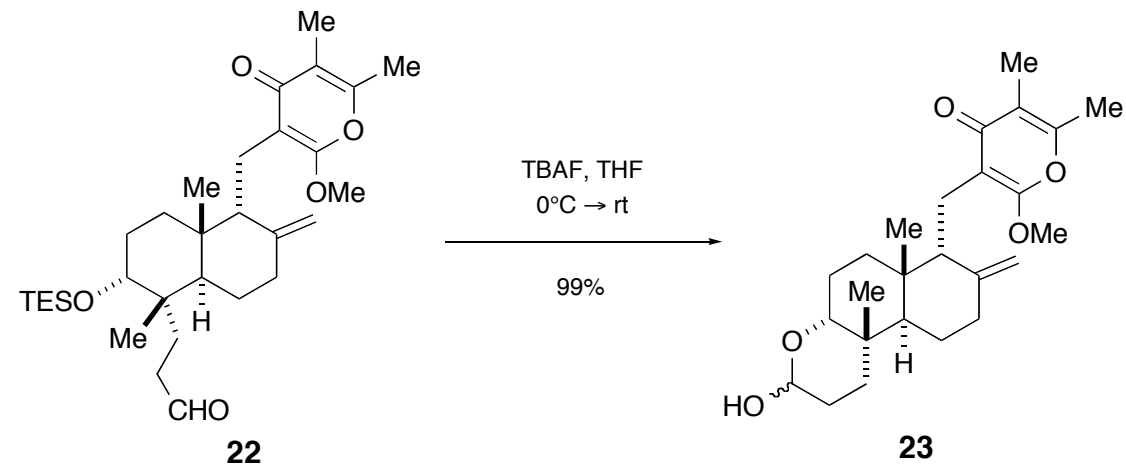

Tetrabutylammonium fluoride (TBAF) in THF (1.0 M solution, $0.150 \mathrm{ml}, 0.15 \mathrm{mmol}$ ) was added dropwise to a stirred solution of $22(7.9 \mathrm{mg}, 15 \mu \mathrm{mol})$ in THF $(1 \mathrm{ml})$ at $0^{\circ} \mathrm{C}$ under argon, and stirring was continued for $40 \mathrm{~min}$ at room temperature. The reaction was quenched with saturated aqueous ammonium chloride $(1 \mathrm{ml})$ at $0^{\circ} \mathrm{C}$, and the resulting mixture 
was extracted with ethyl acetate $(3 \times 10 \mathrm{ml})$. The combined extracts were washed with saturated aqueous sodium hydrogen carbonate $(3 \times 8 \mathrm{ml})$ and brine $(3 \times 8 \mathrm{ml})$, then dried over $\mathrm{Na}_{2} \mathrm{SO}_{4}$. Concentration of the solvent in vacuo afforded a residue, which was purified by column chromatography (hexane-ethyl acetate, $2: 1 \rightarrow 1: 1)$ to give 23 (1:1 diastereomeric mixture) (6.1 $\mathrm{mg}, 99 \%)$ as a colorless viscous liquid. $[\alpha]_{\mathrm{D}}{ }^{20}-18.3^{\circ}\left(c 0.25, \mathrm{CHCl}_{3}\right) ;{ }^{1} \mathrm{H} \mathrm{NMR}\left(500 \mathrm{MHz}, \mathrm{CDCl}_{3}\right) \delta 0.80(3 / 2 \mathrm{H}, \mathrm{s}), 0.84$ $(3 / 2 \mathrm{H}, \mathrm{s}), 0.97$ (3H, s), 0.98-1.03 (1/2H, m), 1.28-1.36 (3H, m), 1.41-1.52 (2H, m), 1.59-1.72 (5/2H, m), 1.88-2.04 (3H, m), $1.90(3 \mathrm{H}, \mathrm{s}), 2.08-2.14(1 / 2 \mathrm{H}, \mathrm{m}), 2.15-2.20(1 / 2 \mathrm{H}, \mathrm{m}), 2.24(3 \mathrm{H}, \mathrm{s}), 2.26-2.38(2 \mathrm{H}, \mathrm{m}), 2.39-2.46(1 / 2 \mathrm{H}, \mathrm{m}), 2.54-2.63(1 \mathrm{H}$, m), 2.68-2.74 (1/2H, m), 2.81 (1/2H, dd, J=4.7, 13.2 Hz), 3.05-3.12 (1/2H, m), 3.35 (1/2H, t, J=2.7 Hz), 3.80-3.84 (1/2H, m), $3.86(3 / 2 \mathrm{H}, \mathrm{s}), 3.87(3 / 2 \mathrm{H}, \mathrm{s}), 4.17-4.20(1 / 2 \mathrm{H}, \mathrm{m}), 4.22-4.25(1 / 2 \mathrm{H}, \mathrm{m}), 4.44-4.56(1 \mathrm{H}, \mathrm{m}), 4.71(1 / 2 \mathrm{H}, \mathrm{br} \mathrm{s}), 5.33(1 / 2 \mathrm{H}, \mathrm{br}$ s); ${ }^{13} \mathrm{C} \mathrm{NMR}\left(125 \mathrm{MHz}, \mathrm{CDCl}_{3}\right) \delta 10.1,14.1,16.9,19.8,22.6,22.9,23.3,24.4,29.1,31.4,33.8,34.5,35.1,37.5,55.3,56.0$, 80.3, 96.9, 103.5, 109.0, 120.1, 149.3, 154.7, 162.7, 180.4; IR (neat) 3403, 2930, 2292, 1726, 1671, 1589, 1462, 1422, 1377, 1321, 1260, 1019, 951, 885, 802, $754 \mathrm{~cm}^{-1}$; HREIMS $(\mathrm{m} / \mathrm{z})$ calcd for $\mathrm{C}_{25} \mathrm{H}_{34} \mathrm{O}_{4}\left[\left(\mathrm{M}-\mathrm{H}_{2} \mathrm{O}\right)^{+}\right], 398.2457$, found 398.2447.

\section{3-[[(4aR,6a $R, 7 R, 10 \mathrm{a} R, 10 \mathrm{~b} R)-6 \mathrm{a}, 10 \mathrm{~b}-D i m e t h y l-8-m e t h y l e n e-4 a, 5,6,6 \mathrm{a}, 7,8,9,10,10 \mathrm{a}, 10 \mathrm{~b}-\mathrm{decahydro}-1 H$-benzo[f]-} chromen-7-yl]methyl]-2-methoxy-5,6-dimethyl-4H-pyran-4-one (candelalide A) (1).

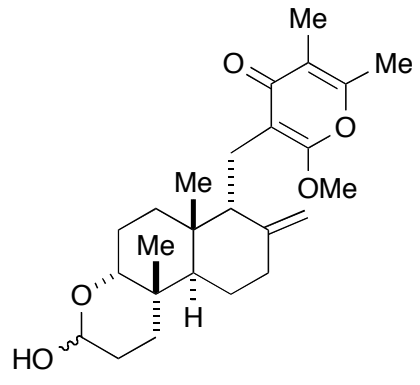

23

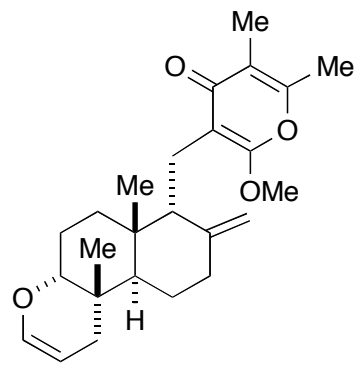

candelalide A (1)

Methanesulfonyl chloride $(1.4 \mu \mathrm{l}, 18 \mu \mathrm{mol})$ was added to a stirred solution of $23(3.0 \mathrm{mg}, 7.2 \mu \mathrm{mol})$ in dry THF (0.5 ml) containing triethylamine $(8.0 \mu \mathrm{l}, 58 \mu \mathrm{mol})$ at $0^{\circ} \mathrm{C}$ under argon, and stirring was continued at room temperature for $1 \mathrm{~h}$. The reaction was quenched with saturated aqueous ammonium chloride $(0.3 \mathrm{ml})$ at $0^{\circ} \mathrm{C}$, and the resulting mixture was extracted with ethyl acetate $(3 \times 8 \mathrm{ml})$. The combined extracts were washed with saturated aqueous sodium hydrogen carbonate $(3 \times 6$ $\mathrm{ml})$ and brine $(3 \times 6 \mathrm{ml})$, then dried over $\mathrm{Na}_{2} \mathrm{SO}_{4}$. Concentration of the solvent in vacuo afforded a residue, which was purified by column chromatography (hexane-ethyl acetate, 1:1) to give $\mathbf{1}(2.9 \mathrm{mg}, 87 \%)$ as a colorless amorphous powder. $[\alpha]_{\mathrm{D}}{ }^{24}-25.1^{\circ}\left(c 0.35, \mathrm{CH}_{3} \mathrm{OH}\right)\left[\mathrm{lit}^{\left[{ }^{[1]}\right.}[\alpha]_{\mathrm{D}}{ }^{24}-23.1^{\circ}\left(c 0.26, \mathrm{CH}_{3} \mathrm{OH}\right)\right] ;{ }^{1} \mathrm{H} \mathrm{NMR}\left(600 \mathrm{MHz}, \mathrm{CDCl}_{3}\right) \delta 0.90(3 \mathrm{H}, \mathrm{s}), 0.99(3 \mathrm{H}, \mathrm{s})$, $1.04(1 \mathrm{H}, \mathrm{td}, J=13.2,3.2 \mathrm{~Hz}), 1.36(1 \mathrm{H}, \mathrm{dq}, J=13.2,4.8 \mathrm{~Hz}), 1.57(1 \mathrm{H}, \mathrm{m}), 1.60(1 \mathrm{H}, \mathrm{m}), 1.75(1 \mathrm{H}, \mathrm{ddd}, J=14.1,6.8,3.6 \mathrm{~Hz})$, $1.85(1 \mathrm{H}, \mathrm{dd}, J=11.7,3.6 \mathrm{~Hz}), 1.89(3 \mathrm{H}, \mathrm{d}, J=0.8 \mathrm{~Hz}), 1.95(1 \mathrm{H}, \mathrm{m}), 1.98(1 \mathrm{H}, \mathrm{dd}, J=11.7,3.0 \mathrm{~Hz}), 2.10(1 \mathrm{H}, \mathrm{dd}, J=17.2,5.5$ Hz), $2.12(1 \mathrm{H}, \mathrm{m}), 2.23(3 \mathrm{H}, \mathrm{s}), 2.37(1 \mathrm{H}, \mathrm{dd}, J=13.6,4.0 \mathrm{~Hz}), 2.42(1 \mathrm{H}, \mathrm{m}), 2.44(1 \mathrm{H}, \mathrm{dd}, J=12.8,11.7 \mathrm{~Hz}), 2.70(1 \mathrm{H}, \mathrm{dd}$, $J=12.8,3.3 \mathrm{~Hz}), 3.71(1 \mathrm{H}, \mathrm{d}, J=1.8 \mathrm{~Hz}), 3.84(3 \mathrm{H}, \mathrm{s}), 4.17(1 \mathrm{H}, \mathrm{dd}, J=2.6,2.2 \mathrm{~Hz}), 4.49(1 \mathrm{H}, \mathrm{dd}, J=2.6,2.5 \mathrm{~Hz}), 4.53(1 \mathrm{H}, \mathrm{dd}$, $J=5.9,2.2 \mathrm{~Hz}), 6.37(1 \mathrm{H}, \mathrm{dd}, J=6.2,2.2 \mathrm{~Hz}) ;{ }^{13} \mathrm{C} \mathrm{NMR}\left(150 \mathrm{MHz}, \mathrm{CDCl}_{3}\right) \delta 10.1,16.9,19.7,22.8,23.4,23.5,23.9,28.7,31.3$, 32.5, 33.6, 34.4, 37.4, 55.3, 56.6, 78.9, 97.6, 103.5, 108.9, 118.5, 142.6, 149.2, 154.7, 162.8, 180.3; IR (neat) 2935, 2361, 1738, 1670, 1591, 1462, 1255, 1145, 1060, 985, 952, $754 \mathrm{~cm}^{-1}$; EIMS (m/z) $398\left(\mathrm{M}^{+}\right), 383,367,355,341,219,167$; HREIMS $(\mathrm{m} / z)$ calcd for $\mathrm{C}_{25} \mathrm{H}_{34} \mathrm{O}_{4}\left(\mathrm{M}^{+}\right)$, 398.2457, found 398.2447. The ${ }^{1} \mathrm{H}$ and ${ }^{13} \mathrm{C} \mathrm{NMR}$, IR, and EIMS spectrum are compatible with those reported in the literature. ${ }^{[1]}$ 